University of Nebraska - Lincoln DigitalCommons@University of Nebraska - Lincoln

U.S. Navy Research

U.S. Department of Defense

2016

\title{
Impact of chevron spacing and asymmetric distribution on supersonic jet acoustics and flow
}

\author{
N. Heeb \\ University of Cincinnati, heebns@mail.uc.edu \\ E. Gutmark \\ University of Cincinnati, gutmarej@ucmail.uc.edu \\ K. Kailasanath \\ Naval Research Laboratory, kailas@lcp.nrl.navy.mil
}

Follow this and additional works at: http:// digitalcommons.unl.edu/usnavyresearch

Heeb, N.; Gutmark, E.; and Kailasanath, K., "Impact of chevron spacing and asymmetric distribution on supersonic jet acoustics and flow" (2016). U.S. Navy Research. 101.

http://digitalcommons.unl.edu/usnavyresearch/101

This Article is brought to you for free and open access by the U.S. Department of Defense at DigitalCommons@University of Nebraska - Lincoln. It has been accepted for inclusion in U.S. Navy Research by an authorized administrator of DigitalCommons@University of Nebraska - Lincoln. 


\title{
Impact of chevron spacing and asymmetric distribution on supersonic jet acoustics and flow
}

\author{
N. Heeb ${ }^{a, *}$, E. Gutmark ${ }^{a}$, K. Kailasanath ${ }^{b}$ \\ a University of Cincinnati, Cincinnati, $\mathrm{OH} 45220$, United States \\ ${ }^{\mathrm{b}}$ Naval Research Laboratory, Washington, DC 20375, United States
}

\section{A R T I C L E I N F O}

\section{Article history:}

Received 30 June 2015

Received in revised form

20 December 2015

Accepted 27 January 2016

Handling Editor: P. Joseph

Available online 11 February 2016

Keywords:

Supersonic Jet Noise

Aeroacoustics

Chevrons

\begin{abstract}
A B S T R A C T
An experimental investigation into the effect of chevron spacing and distribution on supersonic jets was performed. Cross-stream and streamwise particle imaging velocimetry measurements were used to relate flow field modification to sound field changes measured by far-field microphones in the overexpanded, ideally expanded, and underexpanded regimes. Drastic modification of the jet cross-section was achieved by the investigated configurations, with both elliptic and triangular shapes attained downstream. Consequently, screech was nearly eliminated with reductions in the range of 10-25 dB depending on the operating condition. Analysis of the streamwise velocity indicated that both the mean shock spacing and strength were reduced resulting in an increase in the broadband shock associated noise spectral peak frequency and a reduction in the amplitude, respectively. Maximum broadband shock associated noise amplitude reductions were in the 5-7 dB range. Chevron proximity was found to be the primary driver of peak vorticity production, though persistence followed the opposite trend. The integrated streamwise vorticity modulus was found to be correlated with peak large scale turbulent mixing noise reduction, though optimal overall sound pressure level reductions did not necessarily follow due to the shock/fine scale mixing noise sources. Optimal large scale mixing noise reductions were in the $5-6 \mathrm{~dB}$ range.
\end{abstract}

(c) 2016 Elsevier Ltd. All rights reserved.

\section{Introduction}

Application of chevrons to military style nozzles for supersonic jet noise reduction is not a new concept [1-8]. In fact, chevrons are particularly attractive for military aircraft as they can be integrated into the variable area nozzle's divergent flaps which are finite life parts, thus allowing them to be retrofit through regular maintenance. The primary challenge facing chevron introduction into service is maximizing noise reduction during takeoff while limiting the incurred thrust penalties throughout the entire mission. This is particularly difficult as the exhaust of military aircraft at takeoff is highly overexpanded due to nozzle optimization occurring at altitude conditions [6,4]. Not only does the supersonic nature of the exhaust introduce shock associated noise sources, but also it further complicates chevron design due to flow contraction at the nozzle exit caused by the overexpansion. Consequently, larger chevron penetration levels are required to appreciably affect the flow and noise fields $[2,4,5,8]$. This required penetration increase directly raises the overall projected area, a parameter which has been shown to be related to performance losses [1]. One possible route towards mitigation of

\footnotetext{
* Corresponding author.

E-mail addresses: heebns@mail.uc.edu (N. Heeb), gutmarej@ucmail.uc.edu (E. Gutmark), kailas@lcp.nrl.navy.mil (K. Kailasanath).
} 
projected area losses is modification of the other geometric parameters, in particular the chevron's length and width. Consensus on the these parameters has not yet been reached, for example Martens and Spyropoulos [6] and Henderson and Bridges [4] indicate opposing length vs. noise reduction trends. Another possible mitigation tactic is to reduce the number of chevrons while holding their geometry constant. This introduces the parameter of chevron arrangement, in addition to number, towards which limited investigation has been performed. This is primarily due to the faceted design of the variable area exhaust nozzles. In particular, recent studies have limited investigation to sets of 12 chevrons which encompass the entire nozzle perimeter due to the F404's roughly dodecagon nozzle cross section. For example, of the above mentioned studies, Seiner et al. [1] was the only group which did not employ 12 chevrons and only a single chevron configuration was investigated.

Arrangement has been investigated in the past for chevrons applied to non-military style nozzles in both subsonic [9], and supersonic [10-12] jets. Bridges and Brown [9] found that periodic azimuthally spaced chevrons with asymmetric geometry were detrimental to subsonic noise reduction when compared to chevrons with symmetric geometry and identical penetration. Additionally, increases in chevron number were shown to achieve appreciable low frequency reductions without a high frequency penalty. Mengle et al. [10] found that azimuthally varied chevron penetration was beneficial for inflight passenger aircraft shock noise reduction. Additionally, due to a lack of azimuthal periodicity, an azimuthal sound field was measured which required operating condition dependent clocking (relative to the engine's pylon) for optimal noise reduction. Finally, Tide and Srinivasan [11,12] investigated the effect of chevron number and asymmetry on sonic nozzles. They found that increases in chevron number were beneficial, and chevron asymmetries improved noise reduction, in particular broadband shock associated noise (BSAN), compared to standard chevron configurations at specific azimuthal angles. These previous studies illustrate the benefits of investigating arrangement and further highlight the possible shock noise benefits of non-azimuthally symmetric chevron configurations.

The current study seeks to improve understanding of the effect of chevron arrangement on noise from supersonic jets created by military style nozzles. Detailed investigation of the flow and acoustic fields of three chevron arrangements that were conceptualized based on previously investigated noise reduction technologies were performed. The first arrangement was chosen to recreate an elliptic nozzle due to beneficial acoustic results shown in the past. For example, Kinzie et al. [13] and Kinzie and McLaughlin [14,15] found that an elliptic jet was quieter than a corresponding circular jet, with the major axis plane indicating reductions of 4-5 dB. Similar benefits were reported by Verma and Rathakrishnan [16]. Gutmark et al. [17] indicated that elliptic jets reduced the number of shock cells by a factor of three when compared to an equivalent circular jet. Tesson et al. [18] showed that an elliptic jet can reduce broadband shock associated noise by upwards of 3 dB as compared to an equivalent circular nozzle. Generation of an elliptic jet by chevrons was accomplished by clustering two sets of three chevrons at opposite sides of the nozzle. This increased mixing in the plane containing the chevrons due to streamwise vortex introduction, which in turn modified the jet's circular cross-section into an ellipse. The second arrangement was conceptualized to replicate a beveled nozzle. Beveled nozzles are shaped similarly to axisymmetric nozzles except the exit of the nozzle is not perpendicular to the jet centerline. Replication of this configuration was achieved by grouping six chevrons together on one side of the nozzle. Viswanathan [19] showed that a nozzle beveled at $45^{\circ}$ was quieter than a typical nozzle for nearly all observation and azimuthal angles. Maximum overall sound pressure level (OASPL) reductions were approximately $10 \mathrm{~dB}$ and were seen to coincide with the longer lip. Viswanathan et al. [20] investigated the effect of bevel angle and determined that the noise reduction was directly correlated with bevel angle. Measured flow deflection was within $\pm 1.5^{\circ}$ and they documented a maximum of 2.1 simulated EPNdB reduction for a nozzle beveled by $35^{\circ}$. The final chevron arrangement that was investigated was simply the periodically symmetric six chevron configuration. This increases the distance between the chevrons, possibly delaying streamwise vortex interaction and consequently increasing vortex persistence. The arrangement has been shown to be beneficial in the past. For example, Seiner et al. [1] showed that six symmetrically spaced chevrons were able to achieve OASPL reductions of $2 \mathrm{~dB}$ in the peak jet noise direction. In all cases the chevron number was held constant at six to remove the effect of projected area from the investigation. Additionally, due to a modular design which will be discussed below, the exact same chevrons were used for all three configurations, thus limiting discrepancies due to manufacturing processes.

\section{Experimental methods}

\subsection{Facility}

All of the experiments presented within this work were performed in the Aeroacoustic Test Facility (ATF) at the University of Cincinnati. The AFT consists of a $7.3 \mathrm{~m} \times 7.6 \mathrm{~m}$ anechoic chamber, acoustically treated to have a cutoff frequency of $350 \mathrm{~Hz}$, and a coaxial test rig which was offset from the chamber's centerline to increase the available far-field measurement radius. As military style nozzles were being simulated, only the core section of the coaxial rig was used to produce jets. Throughout acoustic testing the secondary flow nozzle was shrouded to eliminate possibility of resonance. The shroud was removed for particle imaging velocimetry (PIV) measurements in an effort to improve ambient tracer particle density following the methodology of Samimy et al. [21] by introduction of seeded low speed secondary flow $(M \approx 0.05$ ). More complete information about the test facility and the test rig can be found in Callender et al. [22]. 


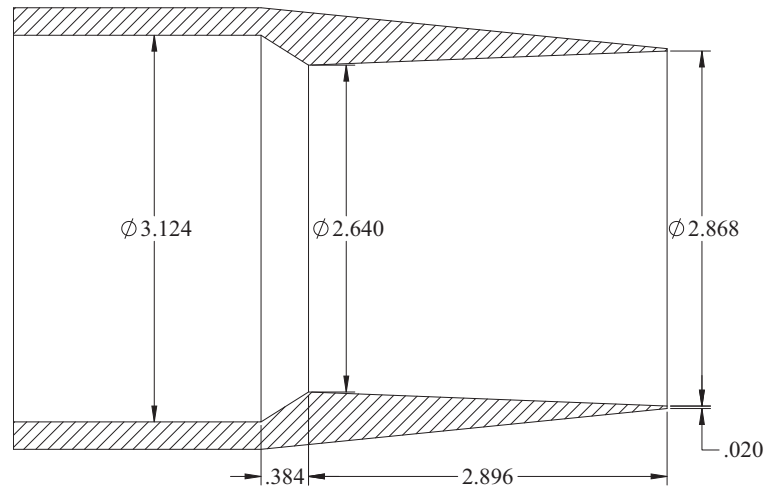

Fig. 1. Baseline nozzle geometry (dimensions in inches).

(a)

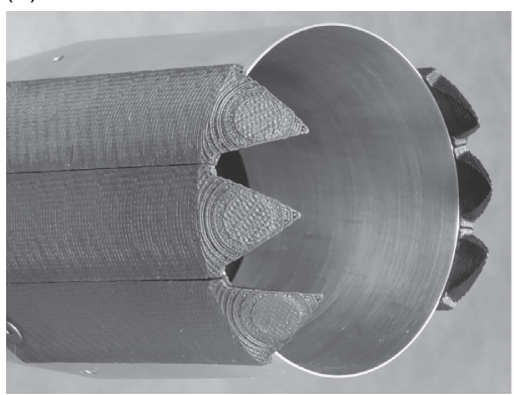

Clustered (b)

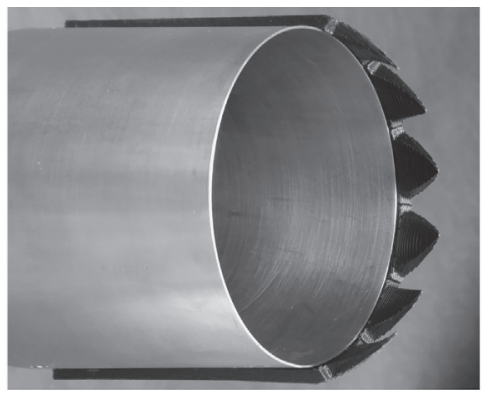

Beveled (c)

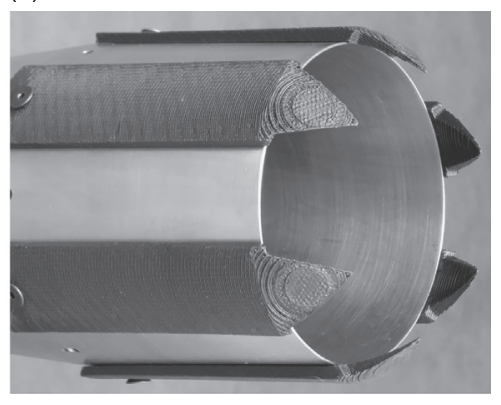

Symmetric

Fig. 2. Chevron arrangements.

\subsection{Model hardware}

\subsubsection{Baseline nozzle}

A biconic converging-diverging nozzle was used in this study to represent a military style variable area nozzle, a schematic of which is shown in Fig. 1. The nozzle has a design Mach number of $M_{d}=1.5$ which corresponds to an area ratio is 1.181. The nozzle's exit diameter, $D_{d}$, is 2.868 in which roughly equates to a $1 / 8$ th scale model. The original investigation of this nozzle was performed by Munday et al. [23] and additional information about the design and flow features can be found therein.

\subsubsection{Chevrons}

To facilitate the measurement of multiple configurations, a set of 12 individual chevrons were created using fused deposition modeling (FDM) rapid prototyping. Twelve corresponding fastener locations were machined into the exterior of the bi-conic nozzle to allow attachment of the chevrons for testing. To limit the number of experiments, three chevron arrangements are selected to be investigated in detail. As noted above, these arrangements were conceptualized based on previously investigated noise reduction technologies.

The first arrangement, shown in Fig. 2a, was created to produce an elliptic jet. This arrangement consisted of two groups of three chevrons assembled towards opposite sides of the nozzle. Due to the layout of the chevrons this configuration was called clustered. The second arrangement, Fig. 2b, was based on a beveled nozzle, such that six chevrons were assembled on one side of the nozzle. Finally, the last arrangement, shown in Fig. 2c, was based on azimuthally symmetry and consisted of six equally spaced chevrons. The use of six chevrons in each of the investigated arrangement was intentional and was done to remove non-constant projected area as an effect on noise as discussed above. For comparison purposes, the configuration consisting of 12 chevrons encompassing the entire nozzle perimeter, denoted here as the standard configuration, was also investigated.

Due to the asymmetry of the beveled and clustered chevron arrangements, the effect of azimuthal angle was investigated. The convention, regarding azimuthal angle, for these configurations is shown in Fig. 3.

Length, penetration, and width are the three main geometric parameters required to define a chevron. Conventionally, penetration is measured off of the lip line (subsonic chevrons), but due to the conical diverging section of the nozzle the definition of penetration is measured as the normal distance between a fictitiously extended nozzle inner contour line and 
(a)

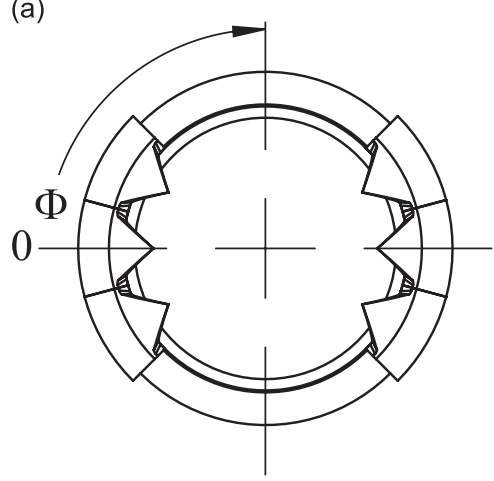

Clustered (b)

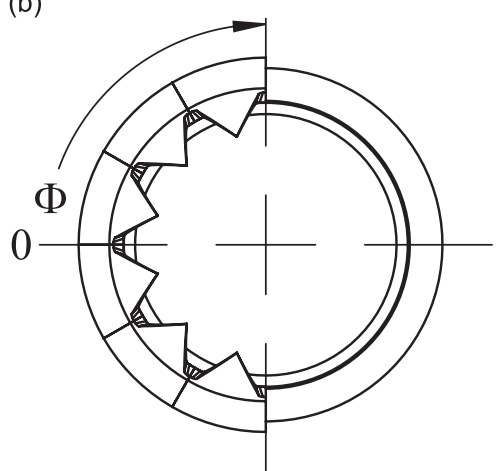

Beveled (c)

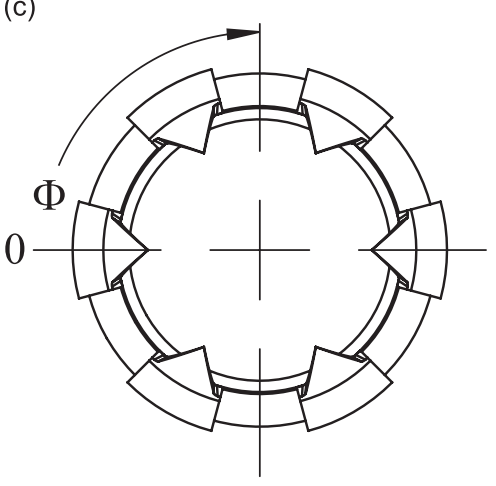

Symmetric

Fig. 3. Conceptual sketch illustrating the convention for the azimuthal angle, $\Phi$.
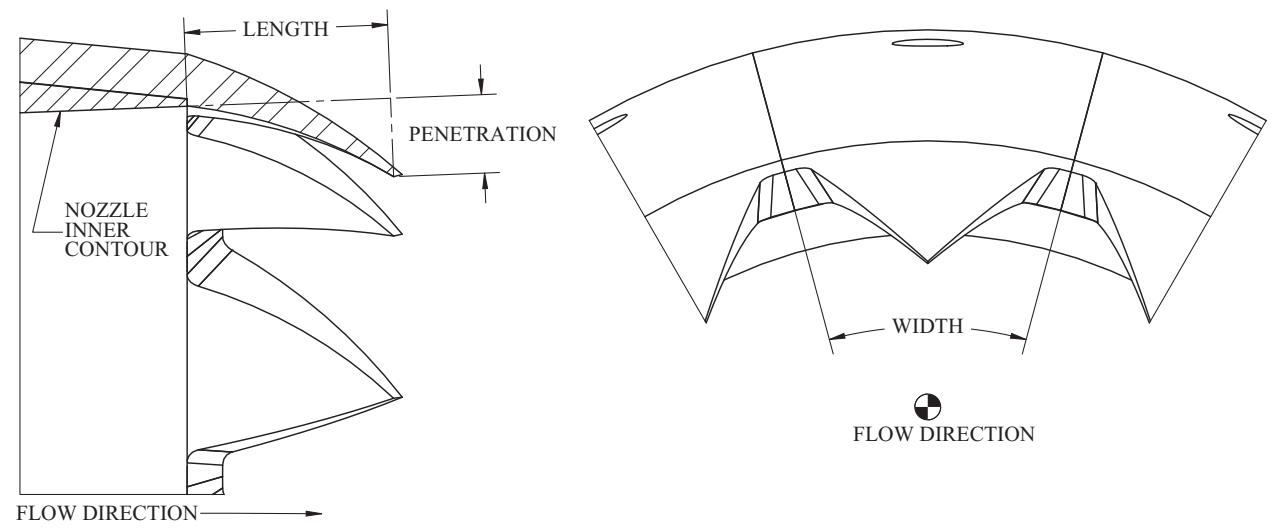

Fig. 4. Definition of chevron geometric parameters.

Table 1

Operating conditions.

\begin{tabular}{llllll}
\hline NPR & NTR & $M_{j}$ & $u_{j}(\mathrm{~m} / \mathrm{s})$ & $D_{j} / D_{d}$ & $R e_{j} / 10^{6}$ \\
\hline 2.50 & 1.00 & 1.22 & 373 & 0.94 & 2.60 \\
3.00 & 1.00 & 1.36 & 403 & 0.96 & 3.17 \\
3.67 & 1.00 & 1.56 & 433 & 1.00 & 3.93 \\
4.00 & 1.00 & 444 & 1.02 & 4.30 \\
\hline
\end{tabular}

the chevron tip. This definition also affects the definition of chevron length, which was altered to be equal to the distance between the nozzle lip and the chevron tip, measured along the fictitiously extended nozzle inner contour. These definitions are described visually in Fig. 4. The chevrons employed in this study had a length, penetration, and width of $0.26 D_{d}, 0.12 D_{d}$, and $30^{\circ}$, respectively. This relatively aggressive design, in terms of penetration (see Heeb et al. [24] for example), was chosen for the current study to ensure that an appreciable effect would occur in a highly overexpanded flow field.

\subsection{Test matrix}

Two overexpanded, the design, and an underexpanded condition were investigated following the work of Munday et al. [25]. Table 1 outlines the chosen conditions along with the fully expanded parameters of Mach number, $M_{j}$, jet velocity, $u_{j}$, Reynolds number, $R e_{j}$, and the fully expanded diameter to design diameter ratio, $D_{j} / D_{d}$. Emphasis will be placed on the design condition $\left(M_{j}=1.50\right)$ and the highly overexpanded condition $\left(M_{j}=1.22\right)$ as they bound typical operation of a military style nozzle.

In an effort to suppress experimental error, measured operating conditions were held with 1.5 percent of target values. These facility parameters were then post-processed following the procedures outlined by the AIAA [26,27] to determine the total uncertainty of each measured and derived quantity. In regards to the measurements presented here, the most 
important parameter, in terms of error, is jet velocity, which was determined with 95 percent confidence to be within $\pm 7.3 \mathrm{~m} / \mathrm{s}$ of the target value.

\subsection{Acoustic measurements}

Thirteen 1/4 in Brüel \& Kjær 4954 microphones, located on a polar array centered on the nozzle exit with a radius of $47 D_{d}$, were used to acquire far-field acoustic measurements. Polar angles were measured from the upstream jet axis. Twelve microphones were located every $10^{\circ}$ between $40^{\circ}$ and $150^{\circ}$. Due to line of sight issues caused by the secondary flow supply, the final microphone was located at $35^{\circ}$, instead of $30^{\circ}$. All acoustic data was taken with the protective microphone grid caps removed to eliminate the need for corrections. Pressure data was acquired for five seconds at approximately $205 \mathrm{kHz}$ and then converted into frequency space following Bartlett's method [28]. The resulting averaged spectrum was then propagated to a radial distance of $100 D_{d}$ with non-standard day atmospheric attenuation corrections applied using the method of Bass et al. [29]. Employing the above mentioned facility uncertainties, Lighthill's eighth power law [30] as well as additional sources of error, the OASPL values represented here were calculated to be within $\pm 0.8 \mathrm{~dB}$ of the true value with 95 percent confidence.

\subsection{PIV measurements}

A LaVision Flow Master system was used to acquire flow field measurements. This system primarily consists of a data acquisition computer, a $120 \mathrm{~mJ}$ dual pulse New Wave Research Nd:Yag laser, and two Imager Intense CCD 1 megapixel 12-bit

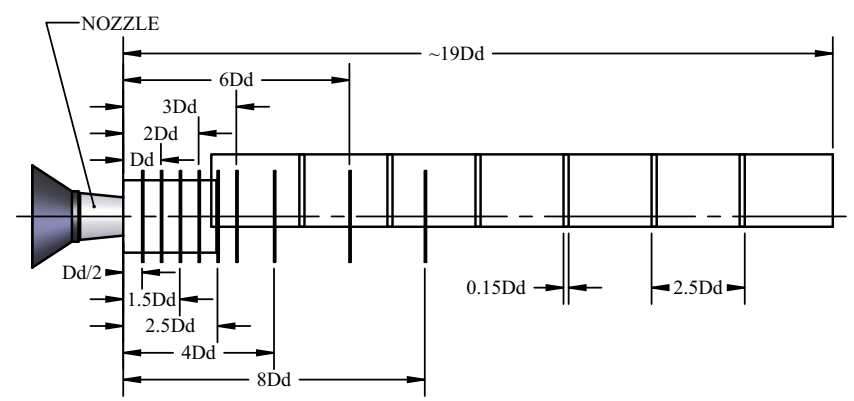

Fig. 5. Details of PIV measurement locations.

(a)

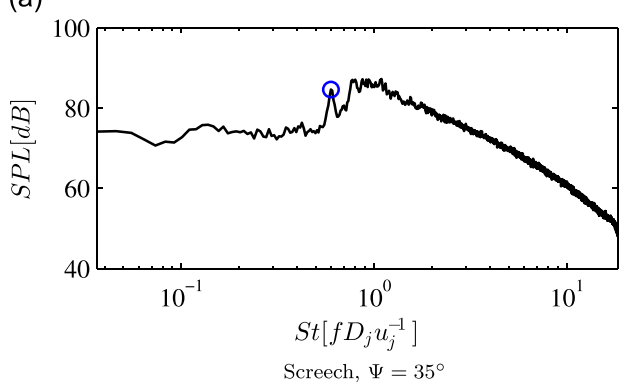

(b)

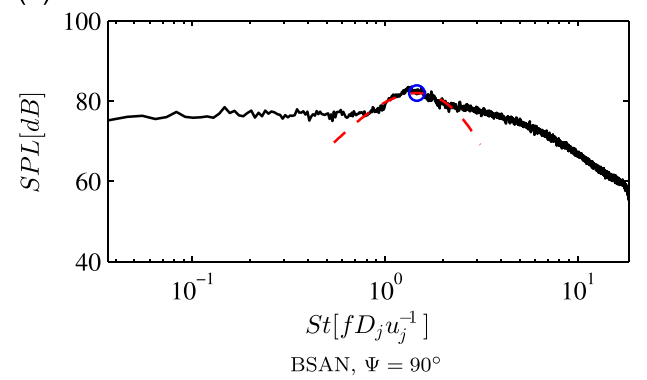

(c)

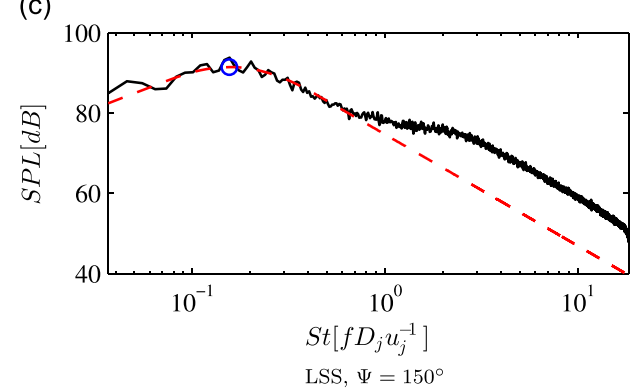

Fig. 6. Illustration of quantitative extraction of noise source metrics. The peak frequency/amplitude is shown as a circle, the dashed lines correspond to pertinent data fit. 
cameras. A cylindrical and spherical lens were used to form a laser plane of desired thickness and width. Additionally, a Nikon lens $(28,35$, or $50 \mathrm{~mm})$, a $532 \mathrm{~nm}$ wavelength filter, and a lens adapter that allowed micro adjustment of the angle at which the lens was positioned were attached to each camera. These adapters allowed corrections to be applied for nonperpendicular viewing of the laser sheet following the Scheimpflug principle. During streamwise measurements the cameras were arranged side-by-side, with a near perpendicular viewing angle. Following the results of Alkislar et al. [31], the cameras were located with a $45^{\circ}$ viewing angle of the laser sheet to reduce measurement error. Both streamwise and cross stream planes were measured, using a two dimensional and stereoscopic PIV configurations, respectively. As the streamwise measurement plane was aligned with the primary flow direction, the laser sheet was selected to be approximately $0.5 \mathrm{~mm}$ thick and a time delay in the range of $5 \mu \mathrm{s}$ was used. The cross stream measurements, on the other hand, experienced reduced particle residence time within the laser sheet. Consequently a laser sheet of approximately 2 mm in thickness and a time delay of $1.5 \mu$ s were used.

The physical extent of the jet column and camera resolution limitations necessitated measurement of multiple planes of data. This was accomplished through use of a three axis traverse system. Four streamwise axial positions and nine cross stream planes were acquired as outlined in Fig. 5. To reduce the number of required axial locations needed to encompass the potential core, only half of the jet was captured during the aft streamwise measurements, which allowed an increase in the axial field of view. In all cases the raw data had a spatial resolution below $2 \mathrm{~mm}$.

Laser masking and a surface treatment consisting of a mat black undercoat and a Rhodamine-B suspension finish coat were used to reduce the effect of hardware reflections on processed data. Several custom built Laskin nozzles were used to atomize olive oil for tracer particles. The corresponding particle sizes were roughly $1 \mu \mathrm{m}$ resulting in a worst case Stoke's number of 0.08 .

LaVision's DaVis software was used for the data analysis. Raw image pairs were pre-processed following the methodology of Deen et al. [32] to reduce the effect of any hardware reflections that were not eliminated using the methods discussed above. A multi-pass method with an initial window size of $64 \times 64$, and a final size of $16 \times 16$ with a 50 percent overlap was used to calculate the velocity field. The results were post-processed though an allowable vector range and finally computation of ensemble statistics.

The flow field results presented within this work will be limited to time average quantities due to the low data rate of the PIV hardware $(5 \mathrm{~Hz})$. From preliminary sets of data it was determined that acceptable convergence of mean results required 500 image pairs near the nozzle $(x / D<5)$ and 1000 downstream. Following the methodology of Carr et al. [33], the precision error of the results was estimated to be 1.2 percent $u_{j}$ and 0.9 percent $u_{j}$ at a 95 percent confidence level for the velocity and RMS velocity, respectively. Incorporation of this result with the methods of Lazar et al. [34] and the iTTC guidelines [35] estimated the total experimental error to be below 7 percent $u_{j}$ for the time average velocity magnitude and less than 15 percent $u_{j}$ for the RMS fluctuating velocity magnitude. This estimate includes facility set point error, precision error, as well as the errors from the PIV setup such as centerline misalignment, camera calibration, and particle lag through shock waves.

Subsets of the above-mentioned operating conditions were selected for the PIV measurements due to limitations of the AFT air system and time constraints. The streamwise measurements consisted of all of the operating conditions excluding the $M_{j}=1.64$ point, while cross stream measurements were only performed for the $M_{j}=1.22$ and 1.50 conditions.

\section{Results}

\subsection{Acoustic field}

Due to the number of configurations and azimuthal angles that were investigated, direct comparison of all available narrowband spectra is difficult. Consequently, the peak amplitude and frequency of three primary noise sources were quantitatively extracted from the acoustic data, an example of which is shown in Fig. 6. A peak locating algorithm was used

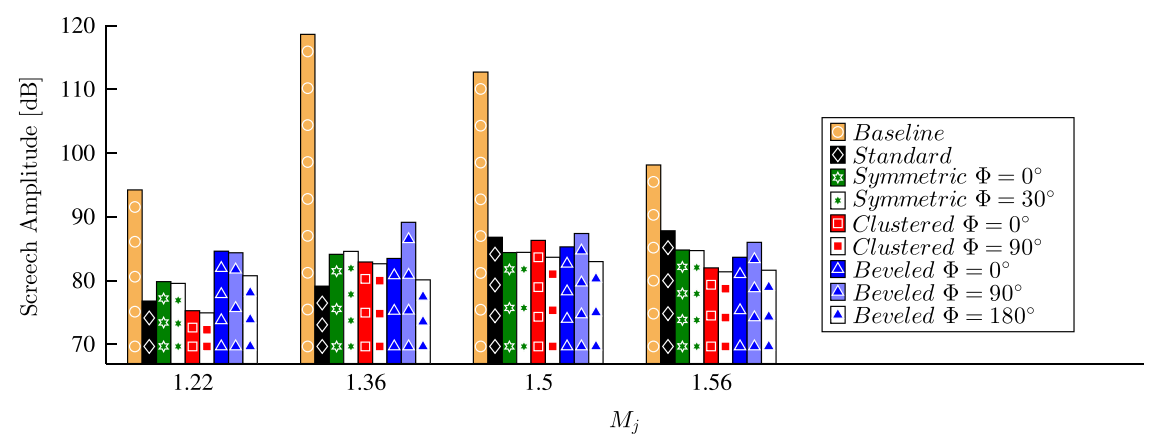

Fig. 7. Measured screech amplitude as a function of $M_{j}$. 
to isolate the primary screech tone from the data acquired at an upstream observation angle of $\Psi=35^{\circ}$, as shown in Fig. 6 a. This observation angle was chosen as the fundamental screech tone primarily propagates upstream due to the weakest link in the aeroacoustic feedback mechanism occurring at the nozzle lip [36]. The BSAN peak frequency and amplitude were determined by curve-fitting a gamma distribution to the acoustic data acquired at $\Psi=90^{\circ}$. This was undertaken so the presence of any screech harmonics would be excluded from peak determination. Additionally, a gamma distribution was chosen over a simple Gaussian as it encompasses the non-symmetric broadband spectral peak [37]. Finally, the large scale structure peak was determined by curve-fitting the large scale similarity spectrum of Tam et al. [38] to the acoustic data acquired at an observation angle of $150^{\circ}$ for Strouhal numbers less than 1 . Due to the presence of broadband shock associated noise in the higher frequency range, extraction of the small scale turbulence noise through use of the fine scale structure similarity spectrum was not possible using the current data set.

Fig. 7 presents the resulting screech amplitudes for all measured operating conditions, configurations, and azimuthal angles. Application of chevrons was able to reduce the screech amplitude in comparison to the baseline jet, independent of azimuthal angle and jet Mach number. An important feature to note is the large amplitude screech observed for the baseline configuration at $M_{j}=1.36$ and 1.50 , which is a feature that has been known to lead to flow field modifications [39,40] as discussed below. Comparison of the azimuthal arrangements to the standard chevron configuration generally shows slightly worse performance in the overexpanded regime and similar or better in the ideally and under expanded conditions. Considering the results of Kastner et al. [41], this is possibly due to a switch in trend regarding the relative centerline Mach disk strength between the standard and azimuthal chevron configurations. Investigation of the effect of azimuthal orientation of the three non-standard configurations on screech reduction indicates that arrangement does play a role in screech reduction. The symmetric configuration shows limited effect as amplitudes were within $0.5 \mathrm{~dB}$ for all investigated operating conditions. This is possibly due to the periodic nature of the configuration. The clustered and beveled configurations on the other hand do show significant azimuthal variation of screech reduction, with the planes not containing chevrons $\left(\Phi=90^{\circ}\right.$ and $\Phi=180^{\circ}$, respectively) achieving optimum reductions. The clustered configuration's $\Phi=90^{\circ}$ plane achieves an average reduction roughly $1 \mathrm{~dB}$ better than the $\Phi=0^{\circ}$ plane, while the beveled configuration's $\Phi=180^{\circ}$ plane outperforms the $\Phi=0^{\circ}$ and $\Phi=90^{\circ}$ planes by over $2.75 \mathrm{~dB}$ and $5.25 \mathrm{~dB}$, respectively. This indicates that the introduction of vorticity within the observation plane is detrimental to screech reduction, possibly due to shock-vortex interaction increasing the overall shock noise amplitude. Reductions are on the order of 10-25 dB for all configurations with the maximum measured reduction just exceeding $38 \mathrm{~dB}$. That said, averaging out the effect of azimuthal orientation and jet Mach number indicated that the clustered configuration achieves an optimum reduction of over $20 \mathrm{~dB}$.

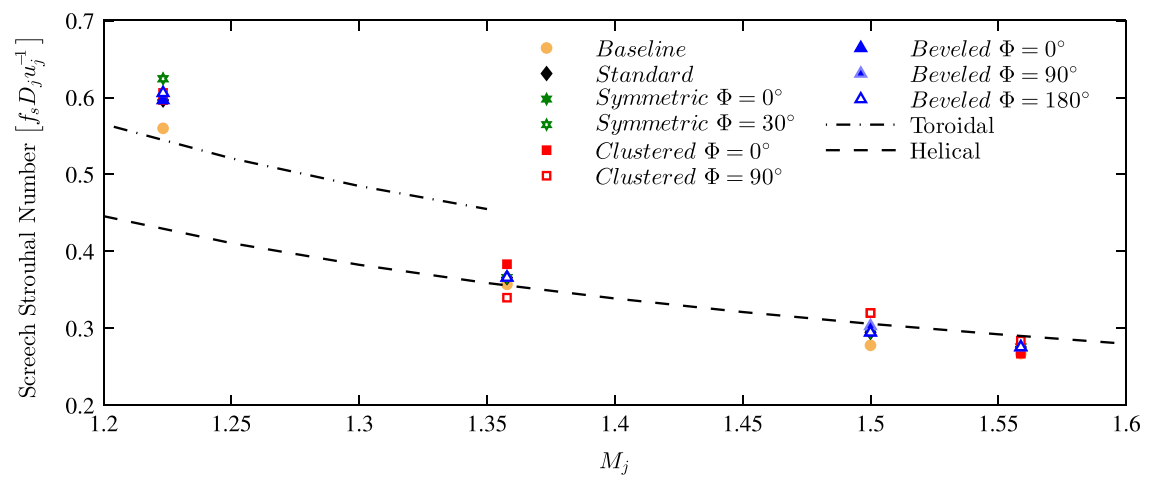

Fig. 8. Measured screech Strouhal number as a function of $M_{j}$. Along with empirical relations of Massey and Ahuja [42].

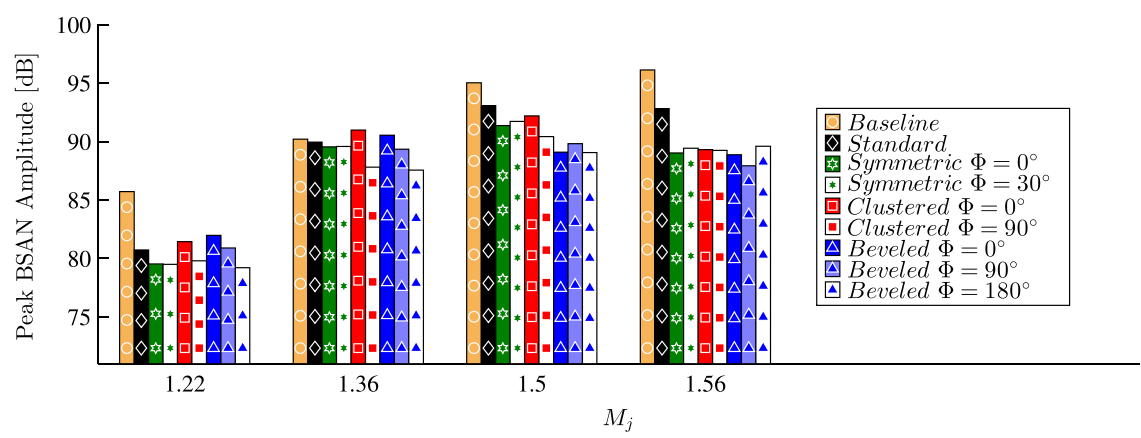

Fig. 9. Measured BSAN peak amplitude as a function of $M_{j} . \Psi=90^{\circ}$. 
The screech frequency of all configurations was also extracted and compared to the empirical relations of Massey and Ahuja [42] in Fig. 8. This relation was selected as it is an empirical extension of the work of Tam et al. [36] (Eq. (1)), which includes the observed effect of instability modes on the screech frequency:

$$
f_{\text {screech }}=\frac{u_{c}}{L_{s}\left(1+M_{c}\right)}
$$

The baseline screech frequency is accurately captured by the prediction for the measured operating range and indicates a transition from helical to toroidal instability for the lowest jet Mach number. Correspondingly, all of the measured screech frequencies were increased at the highly overexpanded $M_{j}=1.22$ condition, confirming the transition to a toroidal mode. Though the screech amplitude was drastically modified by the application of any of the investigated chevron configurations, the measured screech frequencies were relatively unchanged. Similar results have been presented in the past [8,43,44]. It is possible that the standing wave spacing (not measured here) was unchanged, resulting in unchanged screech frequency following the results of Panda [45].

Comparison of the measured BSAN peak amplitudes is presented in Fig. 9. For a majority of the measured azimuthal angles and jet Mach numbers, the application of chevrons reduced the BSAN peak amplitude significantly, though lower reductions or slight increases were observed at the $M_{j}=1.36$ and 1.50 conditions. The under performance at these conditions is thought to be due to the large screech amplitude reductions following the results of Jothi and Srinivasan [46], which indicate that reduction in screech amplitude can lead to amplification of broadband shock associated noise. The typical profile [47-49] is exhibited by all of the configurations investigated; shock noise generally increases with jet Mach number with a local minimum occurring near ideal expansion. The local minimum of the current data is slightly higher than the geometric design condition, a feature which is attributed to spectral amplification due to screech at the design condition as discussed above. Again, the effect of azimuthal orientation of the non-standard configurations can be gleaned from Fig. 9. Similar to the screech results presented above, the symmetric configuration exhibits limited azimuthal variation of the acoustic field, with a maximum difference in peak BSAN less than $0.5 \mathrm{~dB}$. This is thought to result from the symmetry of the configuration. The clustered configuration possess an azimuthal sound field with the $\Phi=90^{\circ}$ plane achieving lower BSAN peak amplitudes than the $\Phi=0^{\circ}$ plane, though the difference in the underexpanded regime is small. The BSAN difference, as computed by averaging across operating condition, exceeds $1.75 \mathrm{~dB}$. Similarly, the beveled configuration's sound field is azimuthal, with the $\Phi=180^{\circ}$ orientation out performing the $\Phi=0^{\circ}$ and $90^{\circ}$ planes at the lower operating conditions. Additionally, a trend of decreasing BSAN amplitude with azimuthal angle exists in the overexpanded range for the beveled configuration. This trend is not exhibited higher in the operating range due to a change in the shock structure as will be discussed below. Similar to the screech discussion above, the optimum azimuthal orientation for the beveled and clustered configurations typically occurs at planes not containing vortex introduction. Consequently, the interaction between the

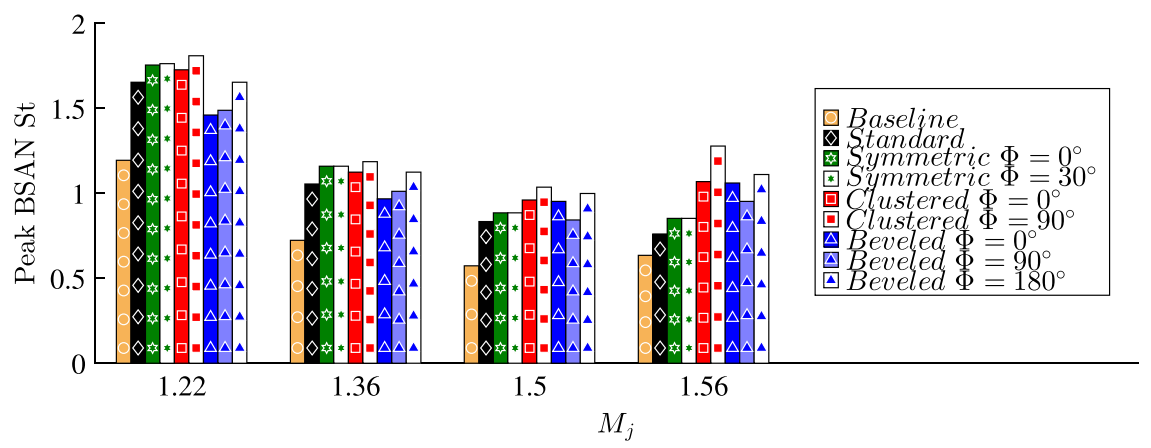

Fig. 10. Measured BSAN peak frequency as a function of $M_{j} . \Psi=90^{\circ}$.

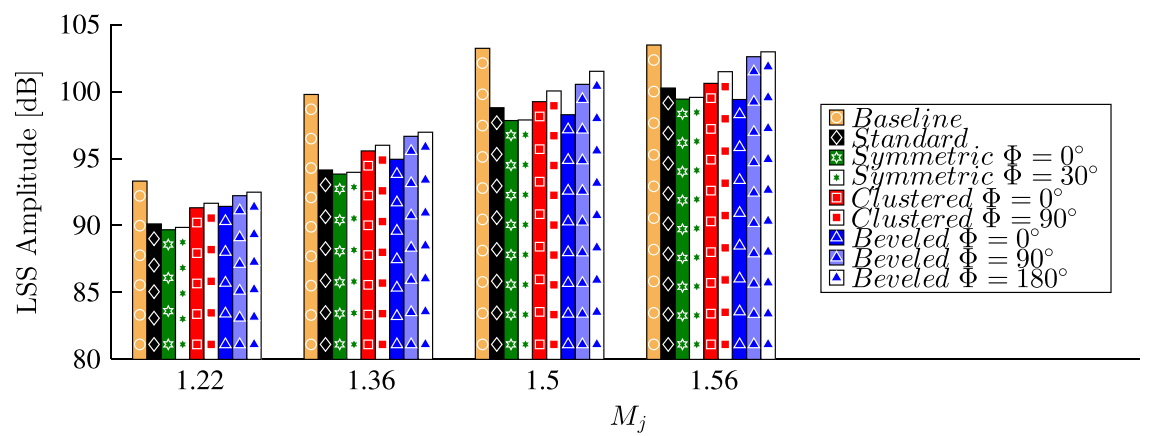

Fig. 11. Measured LSS peak amplitude as a function of $M_{j} . \Psi=150^{\circ}$. 
shock cell structure and streamwise vorticity generated by the chevrons is thought to introduce additional shock associated noise which leads to decreased reductions. Comparison of the standard configuration to the azimuthal arrangements indicated improved reductions in the ideally and under expanded regimes by the three investigated configurations independent of azimuthal orientation. In the overexpanded range, the optimum azimuthal orientation of the symmetric, clustered, and beveled configuration also out performs the standard configuration in terms of peak BSAN reductions. These results are directly related to the average shock cell strength excluding the initially non-quasi-periodic structure following the results of Tam [50] as will be shown below. In general the beveled configuration performs optimally on an azimuthally averaged basis, with reductions in peak BSAN amplitude exceeding $5 \mathrm{~dB}, 1 \mathrm{~dB}, 5.7 \mathrm{~dB}$, and $7.3 \mathrm{~dB}$ at the jet Mach numbers of $1.22,1.36,1.50,1.56$, respectively.

The BSAN peak frequencies corresponding to the amplitudes presented above are shown in Fig. 10. The predominant feature of the results is a universal increase in peak frequency by the application of any chevron configuration. Considering the relation of Tam et al. [36] (shown below in Eq. (2)) this can be explained by a reduction of the average shock cell spacing and/or an increase in the convective velocity, though previous studies $[12,51,44]$ indicate that the reduction in the shock spacing alone can account for the frequency increase:

$$
f_{\mathrm{BSAN}}=\left[L_{s}\left(\frac{1}{u_{c}}+\frac{\cos \Psi}{a_{\infty}}\right)\right]^{-1}
$$

Following the above discussion, the symmetric configuration displayed limited azimuthal variation in peak BSAN frequency. Azimuthal variation exists for the clustered and beveled configurations, with the $\Psi=90^{\circ}$ and $180^{\circ}$ angles consistently achieving the highest peak frequency, respectively. This is expected to be a result of an azimuthally asymmetric shock cell structure resulting from both of the chevron configurations, as will be investigated below. Overall, the $\Phi=90^{\circ}$ clustered configuration achieved the highest BSAN peak frequency, which potentially indicates that this configuration will have the shortest shock cell spacing.

Modification of the large scale mixing noise amplitude as a function of fully expanded jet Mach number is presented in Fig. 11. As with the shock associated noise discussed above, application of any chevron configuration reduced the large scale mixing noise. For the investigated operating conditions, the symmetric configuration out performed all others, though the standard and the beveled configuration at $\Phi=0^{\circ}$ performed similarly in the overexpanded and ideally/underexpanded

(a)

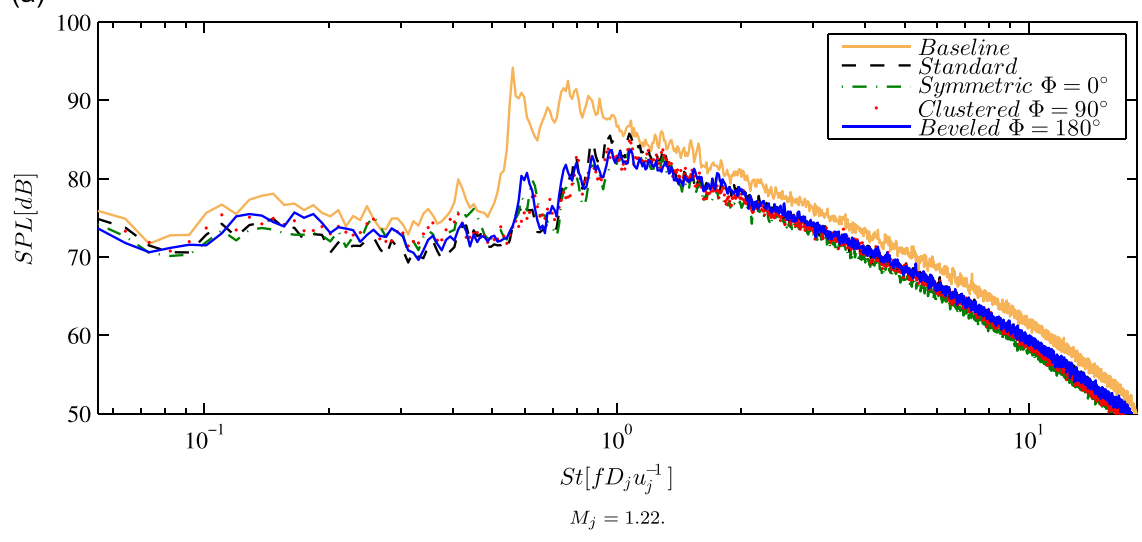

(b)

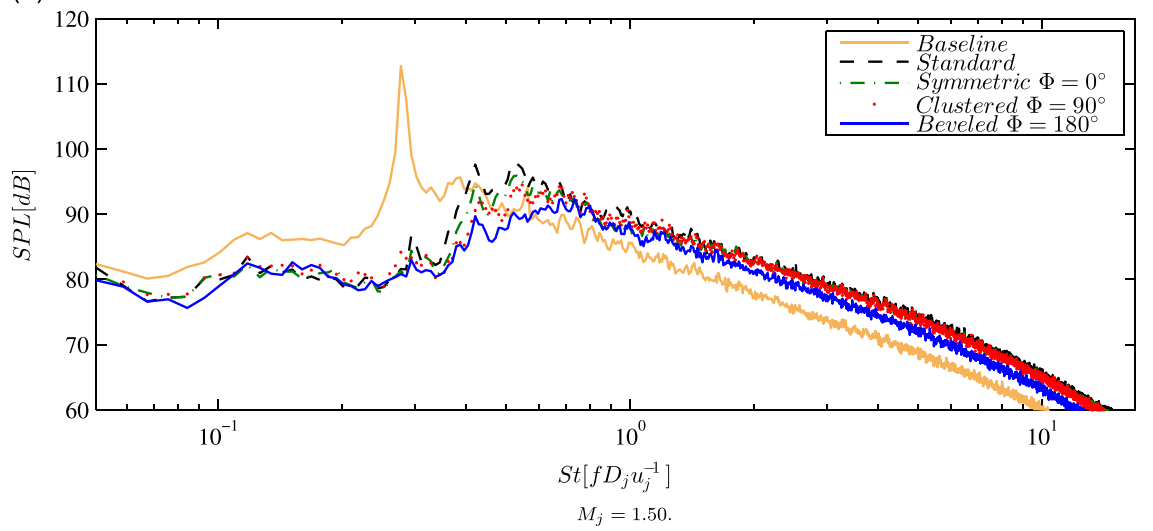

Fig. 12. Spectral comparison detailing effects on screech. $\Psi=35^{\circ}$. Azimuthal orientations selected based on above discussion. 
(a)

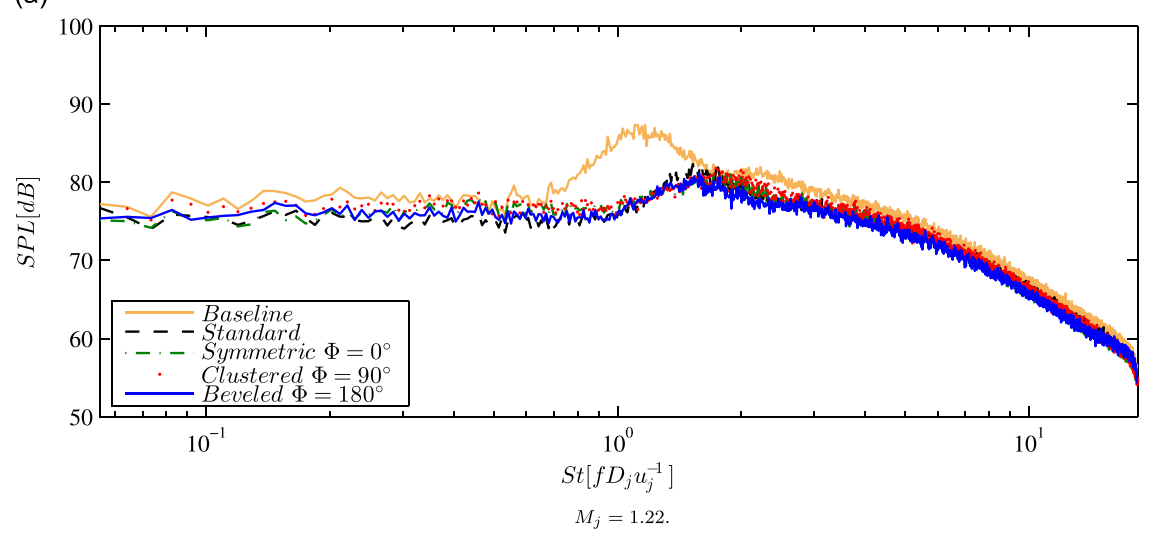

(b)

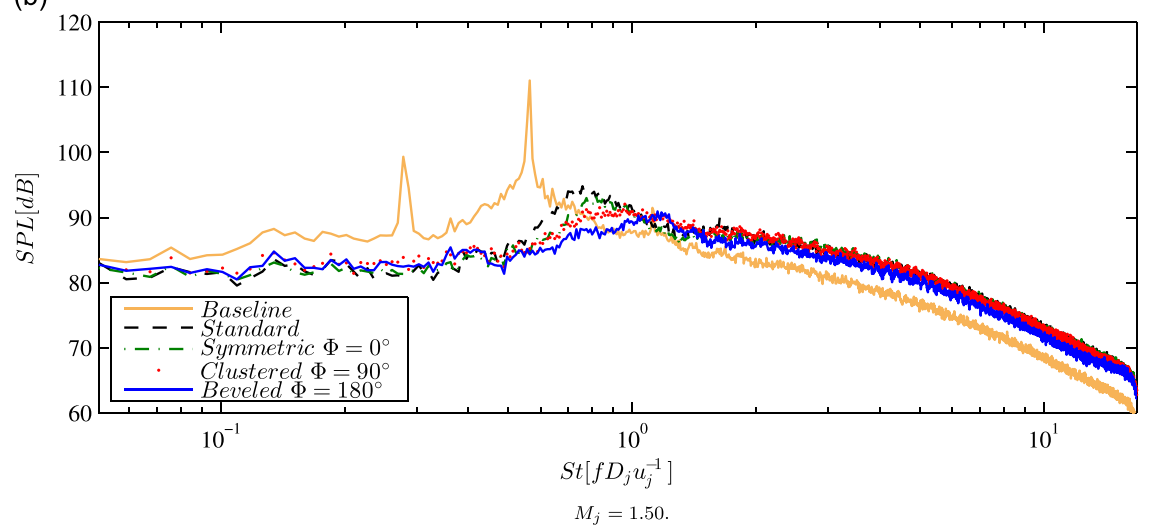

Fig. 13. Spectral comparison detailing effects on BSAN. $\Psi=90^{\circ}$. Azimuthal orientations selected based on above discussion.

regime, respectively. Interestingly, an azimuthal trend, independent of jet Mach number, existed for the symmetric, clustered, and beveled configurations. That is, the orientation in-plane with the chevrons displayed lower large scale structure mixing noise than the other investigated azimuthal angles. This indicates that the presence of the chevrons, and consequently, the induced vorticity reduced large scale mixing noise, most likely due to inhibited near nozzle large scale structure production [52-54]. The beveled configuration showed the largest azimuthal variation in large scale mixing noise amplitude, with the $\Phi=180^{\circ}$ orientation tending toward the baseline values (within $1 \mathrm{~dB}$ ) at operating conditions not displaying large amplitude screech. This reinforces the previous statement regarding the necessity of localized streamwise vorticity for large scale mixing noise reduction. Overall, large scale mixing noise reductions were typically in the range of 3-5 $\mathrm{dB}$, though the symmetric configuration was able to exceed $6 \mathrm{dBs}$ of reduction at the $M_{j}=1.36$ and 1.50 conditions.

Finally, spectral comparisons of the identified optimum azimuthal orientations for peak screech, broadband shock associated noise, and large scale turbulent mixing noise reductions are presented in Figs. 12, 13, and 14, respectively. As with the analysis performed above, screech was investigated at $\Psi=35^{\circ}$, BSAN at $\Psi=90^{\circ}$, and the turbulent mixing noise at $\Psi=150^{\circ}$ due to preferred propagation direction.

In addition to the large amplitude screech reduction, the chevron configurations significantly modified the high frequency content of the upstream propagating spectrum. At the overexpanded condition $\left(M_{j}=1.22\right)$, reductions were achieved across the entire frequency range, with decreases exceeding $12 \mathrm{~dB}$ at the baseline's BSAN peak and a roughly constant decrease of $2 \mathrm{~dB}$ at Strouhal numbers greater than 1. The increase in peak BSAN frequency due to the application of the chevron configurations was also observable at the $\Psi=35^{\circ}$ observation angle, and was the primary cause of the large SPL reduction at the baseline's peak BSAN frequency. Overall, the symmetric configuration achieved the lowest OASPL, with a reduction exceeding $5.75 \mathrm{~dB}$ as compared to the baseline. The ideally expanded results also displayed increased BSAN frequency due to the chevrons, with increases in the 3-4 dB range for Strouhal numbers greater than 1 . This is similar to the discussion presented by Tam and Zaman [55] in regards to high frequency increase of subsonic jets due to application of chevrons. Normalization of the acoustic spectrum using the Helmholtz number instead of the Strouhal number (not shown) confirmed that the SPL increase at high frequency is a result of a shift due to shortened shock cells. The observed large high frequency increases are counter balanced by reductions made at low frequencies resulting in OASPL levels that are lower than that of the baseline. The beveled configuration achieves the highest OASPL reduction at just under $3.5 \mathrm{~dB}$, primarily due to relative decreases in BSAN noise at Strouhal numbers greater than 0.2, as compared to the other chevron configurations. 
(a)

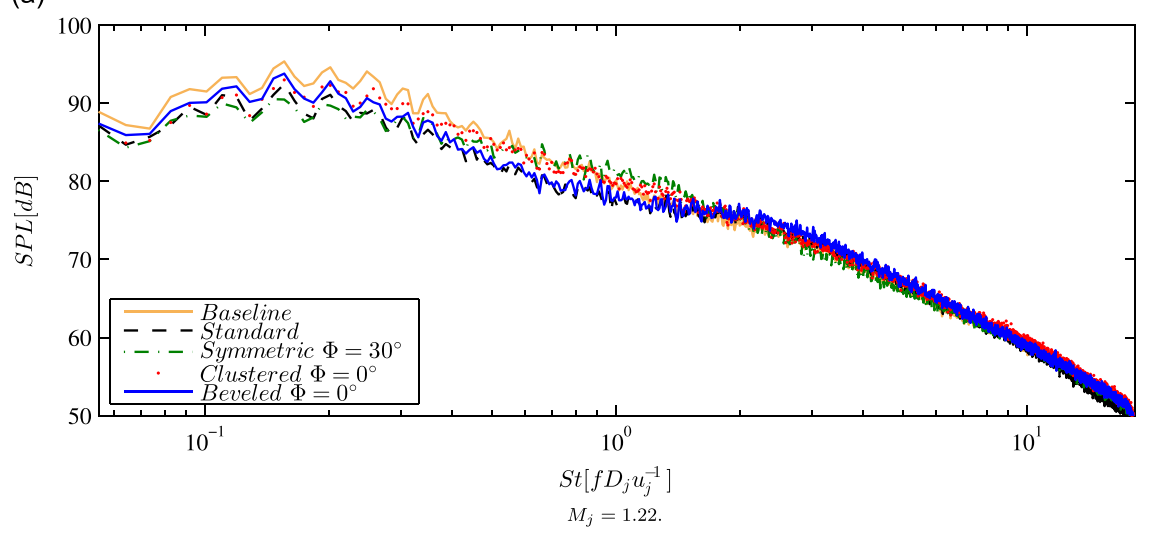

(b)

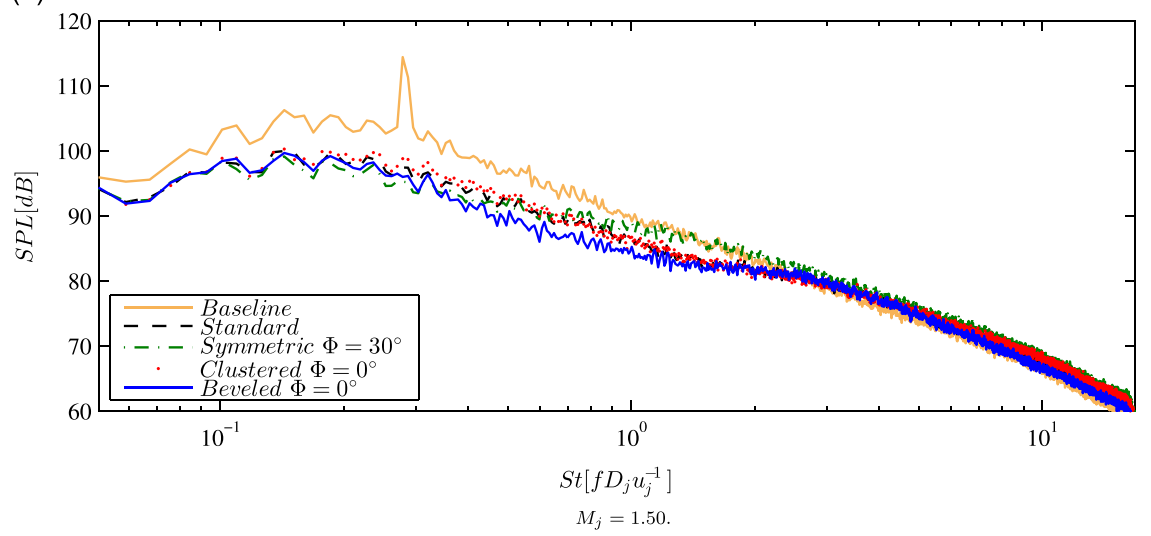

Fig. 14. Spectral comparison detailing effects on turbulent mixing noise. $\Psi=150^{\circ}$. Azimuthal orientations selected based on above discussion.

Similar to the discussion above, the frequency shift of the BSAN resulted in significant modification of the high frequency content of the baseline's acoustic spectrum at $\Psi=90^{\circ}$. Again, acoustic reduction was achieved at all frequencies at the overexpanded condition, independent of the investigated chevron configuration. Reductions at the baseline's peak BSAN exceed $9 \mathrm{~dB}$, while the higher frequencies were reduced on average by $1 \mathrm{~dB}$. The beveled configuration again achieved the optimum reductions as compared to the baseline which just exceed $2.5 \mathrm{~dB}$. The decrease in reduction as compared to $\Psi=35^{\circ}$ was primarily due to the lack of screech propagation toward the $90^{\circ}$ direction at this operating condition. The ideally expanded results indicated a high frequency increase of roughly $4 \mathrm{~dB}$ which was confirmed to be a result of the frequency shift following nondimensionalization by the Helmholtz number. Due to the high amplitude screech noted above, tones occurred even at the sideline observation angle for the baseline configuration. Consequently, reductions on the order of $20 \mathrm{~dB}$ are achieved at specific frequencies. Furthermore, the beveled configuration was optimum and was able to achieve OASPL reductions approximately equal to the $\Phi=35^{\circ}$ results discussed above.

Lastly, the primary effect of the investigated chevron configurations on turbulent mixing noise was the reduction of the large scale mixing noise discussed above. Fig. 14 indicates that limited SPL increases at Strouhal numbers greater than 2 . In the past, chevrons have been shown to increase small scale mixing noise as a result of increased near nozzle small scale turbulence production in both the subsonic and supersonic regimes [56,8]. Consequently, any observed increases in high frequency noise at $\Phi=150^{\circ}$ is a combined result of the increase of BSAN frequency and this redistribution of large scale structures into smaller scales [5]. Differences in the higher frequency content lead the optimum overall noise reducing configuration to differ from the peak large scale mixing noise optimum determined above in Fig. 11. This is particularly evident for the symmetric configuration in the $0.4<S t<3$ range. The standard configuration optimally reduced the OASPL $(2.8 \mathrm{~dB})$ at the overexpanded condition, while the beveled configuration achieved the highest reduction of $7.1 \mathrm{~dB}$ at the design condition.

\subsection{Flow field}

Detailed PIV measurements were acquired in an effort to determine the effect of chevron arrangement on a supersonic jet's flow field. To measure flow features aligned with and perpendicular to the jet axis, both streamwise and cross stream planes were collected. In an effort to capture the entire potential core, the streamwise measurements encapsulated roughly 
(a)

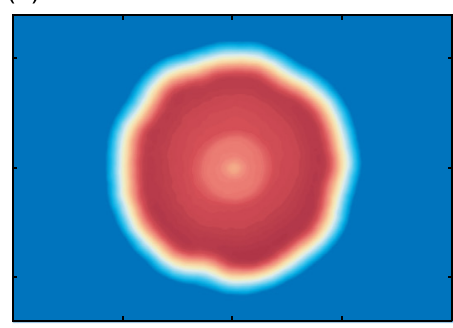

Baseline, $z / D_{j}=1.0$

(d)

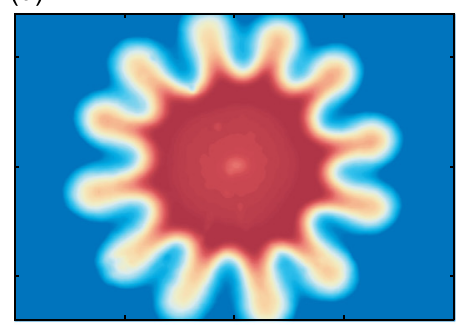

Standard, $z / D_{j}=1.0$.

(g)

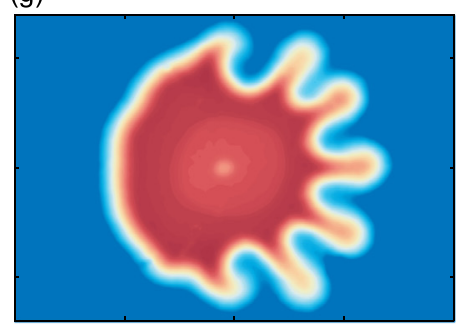

Beveled, $z / D_{j}=1.0$.

(j)

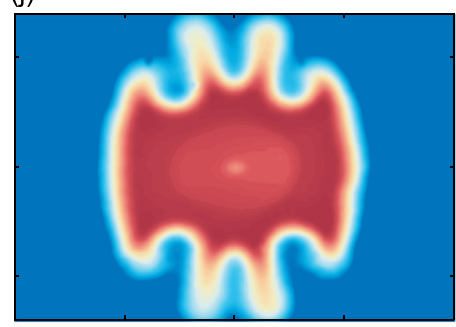

Clustered, $z / D_{j}=1.0$.

(m)

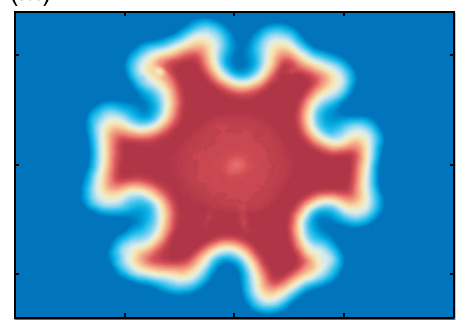

Symmetric, $z / D_{j}=1.0$. (b)

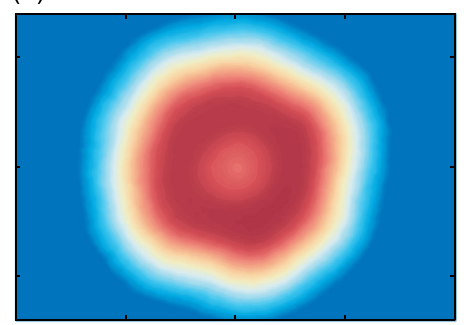

Baseline, $z / D_{j}=3.0$.

(e)

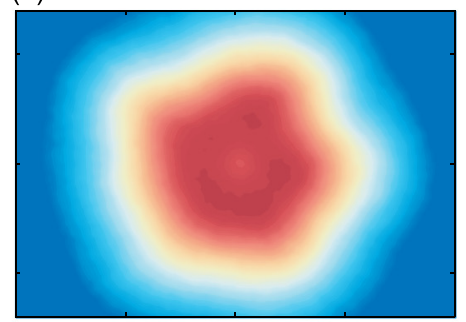

Standard, $z / D_{j}=3.0$.

(h)

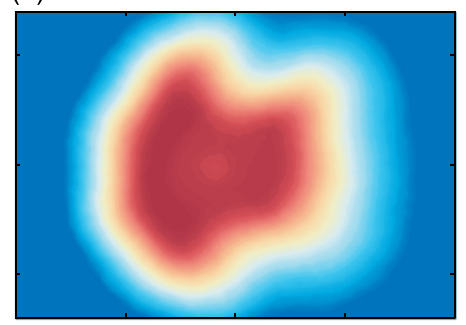

Beveled, $z / D_{j}=3.0$.

(k)

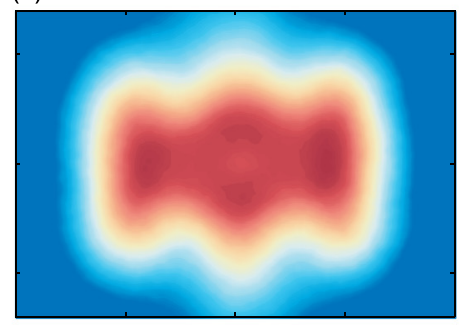

Clustered, $z / D_{j}=3.0$.

(n)

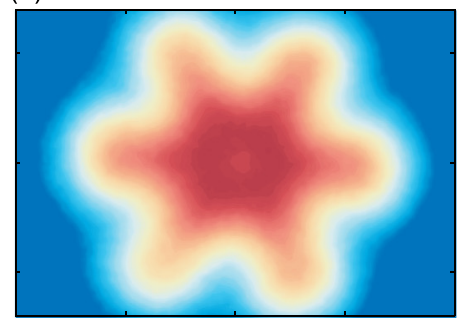

Symmetric, $z / D_{j}=3.0$. (c)

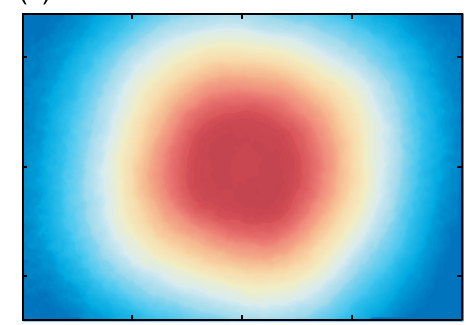

Baseline, $z / D_{j}=6.0$.

(f)

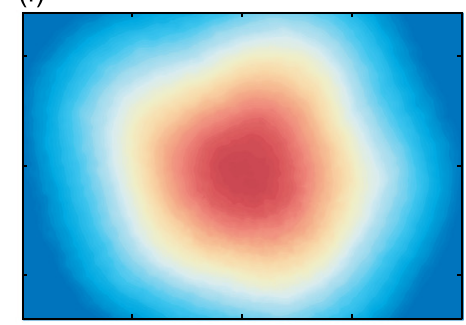

Standard, $z / D_{j}=6.0$.

(i)

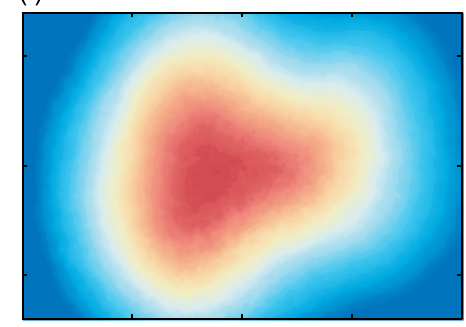

Beveled, $z / D_{j}=6.0$.

(I)

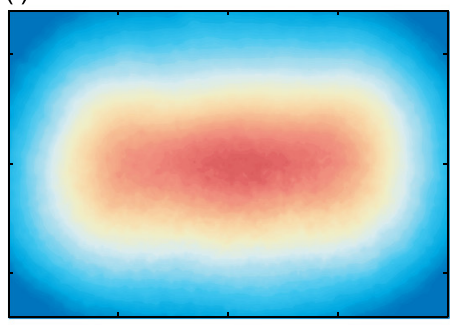

Clustered, $z / D_{j}=6.0$.

(0)

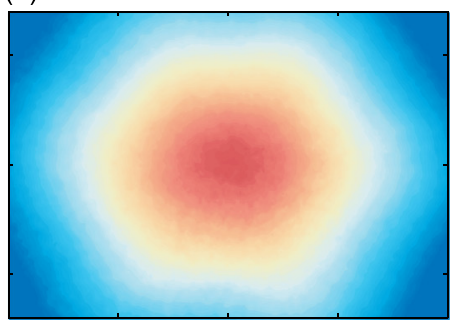

Symmetric, $z / D_{j}=6.0$.

Fig. 15. Evolution of jet cross section with downstream position. $M_{j}=1.22$. 
$19 D_{d}$, while the cross stream measurements were clustered near the nozzle exit due to the rapid decay of streamwise vorticity.

Modification of the jet cross section is best assessed through comparison of the spatial evolution of cross stream planes as shown in Fig. 15 for the overexpanded $\left(M_{j}=1.22\right)$ condition. The sub-figures are arranged such that axial location is constant in each column and increases from left to right while configuration is constant across the rows. The baseline results indicated a nearly axisymmetric jet, with slight deviations from circular around the periphery of the shear layer. This was due to inconsistent surface finish due to application of Rhodamine paint, similar to that shown by Alkislar et al. [57]. Application of any chevron configuration drastically modified the initial jet development. The standard chevron configuration initiated a lobed structure with 12 jetlets [55] that evolved and reduced with downstream propagation, but the effect of which was still discernible 6 diameters downstream. The beveled configuration initiated a similar structure in the quadrant with chevrons, while the unmodified edge was roughly circular. As the jet propagated downstream the cross sectional shape evolved into a roughly triangular shape, the gross effect of which was evident further downstream than the modifications by the standard configuration. The clustered configuration introduced vortices at opposite sides of the jet, which resulted in accelerated mixing in those quadrants. This in turn, caused the jet to develop an elliptic cross section downstream which, similar to the beveled configuration, persisted further downstream than the standard configuration. Finally, the symmetric configuration introduced a six lobed structure very similar to that presented by Seiner et al. [1]. Due to the increased radial spacing between the chevrons, a reduction in negative vortex interaction occurred, allowing the cross-sectional modification to persist further downstream than the standard configuration, as seen at $z / D_{d}=3.0$. As evident by the cross-sectional modifications, the typical under performance of chevrons in the highly overexpanded regime $[5,8,24]$ was not an issue in the current study due to the use of relatively high penetration chevrons.

To further illustrate the evolution of jet cross section, Fig. 16 was generated by extracting the jet half-width from each of the available cross sections and superimposing them together for each individual configuration. As expected from the contours presented above, the baseline flow field was roughly axisymmetric. Investigation of the standard configuration's cross-sections indicated that the radial growth of the jetlets peaked at 1 diameter downstream. Further along the jet column the effect of turbulence reduced the individual jetlet structure and by 2.5 diameters downstream the sinusoidal profile was eliminated. The jetlets of the beveled and clustered configurations were of similar shape to the standard configuration due to the chevron grouping, and consequently showed a similar peak in jetlet growth at 1 diameter downstream. On the other hand, the symmetric configuration did not possess a similar jetlet structure as the reduction in chevron proximity eliminated the interaction between neighboring vortex pairs responsible for large fluid expulsion in the other configurations. Further downstream, the cross-sectional shape of all of the configurations was smoothed by mixing and only large scale modifications remained. Due to the rotational symmetry of the standard and symmetric configurations, the downstream cross-sectional shapes tended toward axisymmetric, while the beveled and clustered configurations took on triangular and elliptic cross sections, respectively, due to their specific azimuthal arrangements. For the three non-standard chevron

(a)

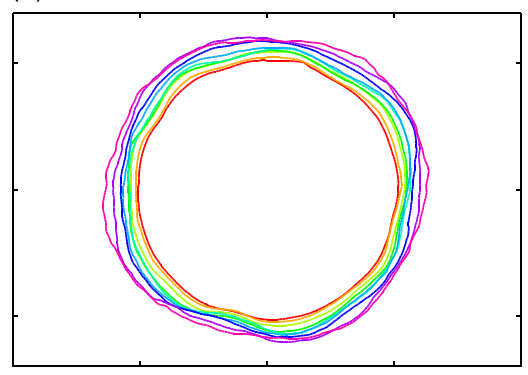

Baseline.

(d)

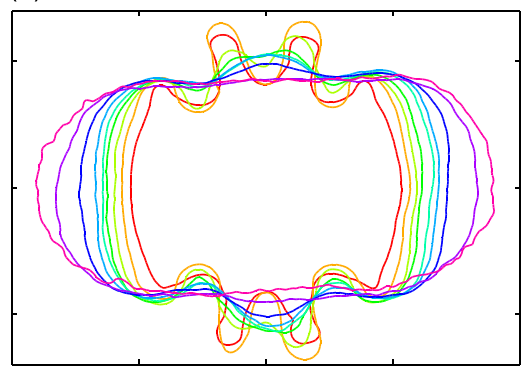

Clustered. (b)

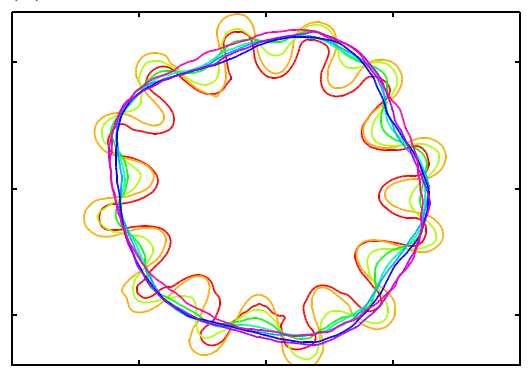

Standard.

(e)

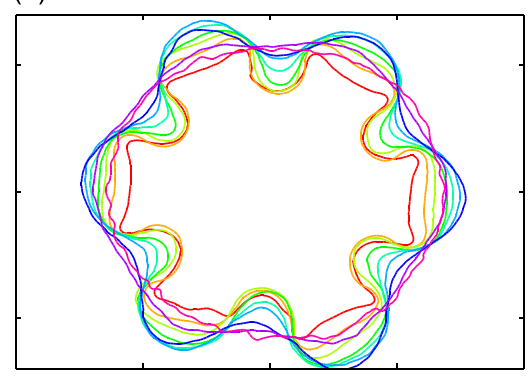

Symmetric. (c)

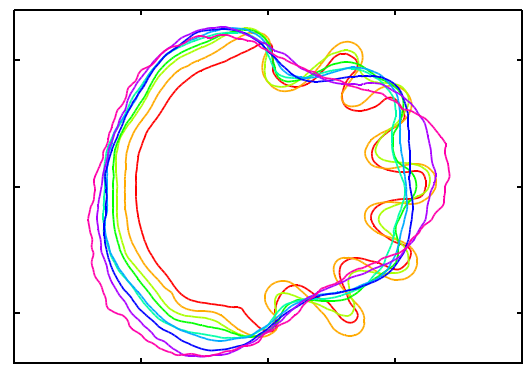

Beveled.

(f)

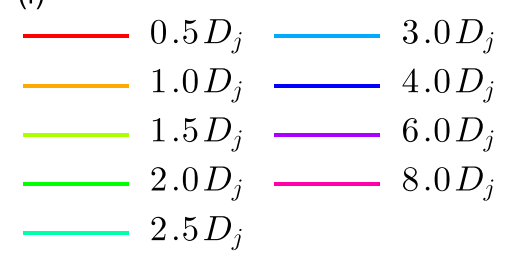

Fig. 16. Downstream evolution of jet cross-section shown by lines of constant velocity, $V=1 / 2 V_{j} . M_{j}=1.22$. 
configurations these downstream shapes were achieved by roughly 6 diameters downstream, while the standard configuration achieved the roughly axisymmetric shape by approximately 3 diameters downstream, indicating possible negative vortex interaction.

As modification of axial jet features, such as the shock cell structure, are not easily determined from cross stream measurements, streamwise contours of time mean velocity were acquired as presented in Fig. 17. These figures are divided in half to ease comparison of the pertinent azimuthal planes and comprise the entirety of the streamwise measured flow field. A diagram of the orientation of the specific measurement plane is also provided within each half of the figure to clearly illustrate the orientation of the configuration. Additionally, as a majority of the jet modifications occurred in the near nozzle region, enlarged views are presented in Figs. 18 and 19 for the over and ideally expanded conditions, respectively.

The baseline jet flow field possess the large Mach disk and corresponding slip lines near the jet axis typical of overexpanded jets from conical CD nozzles [24,25,58], which are particularly apparent in Fig. 18a. Additionally, though the baseline jet exhausts from a conical nozzle, the typical double diamond shock cell structure was not present at $M_{j}=1.22$, similar to the results shown by Munday et al. [25]. The standard configuration reduced the shock cell spacing and the centerline Mach disk strength, while the initial spread rate and slip line radius was increased. Interestingly, the shock
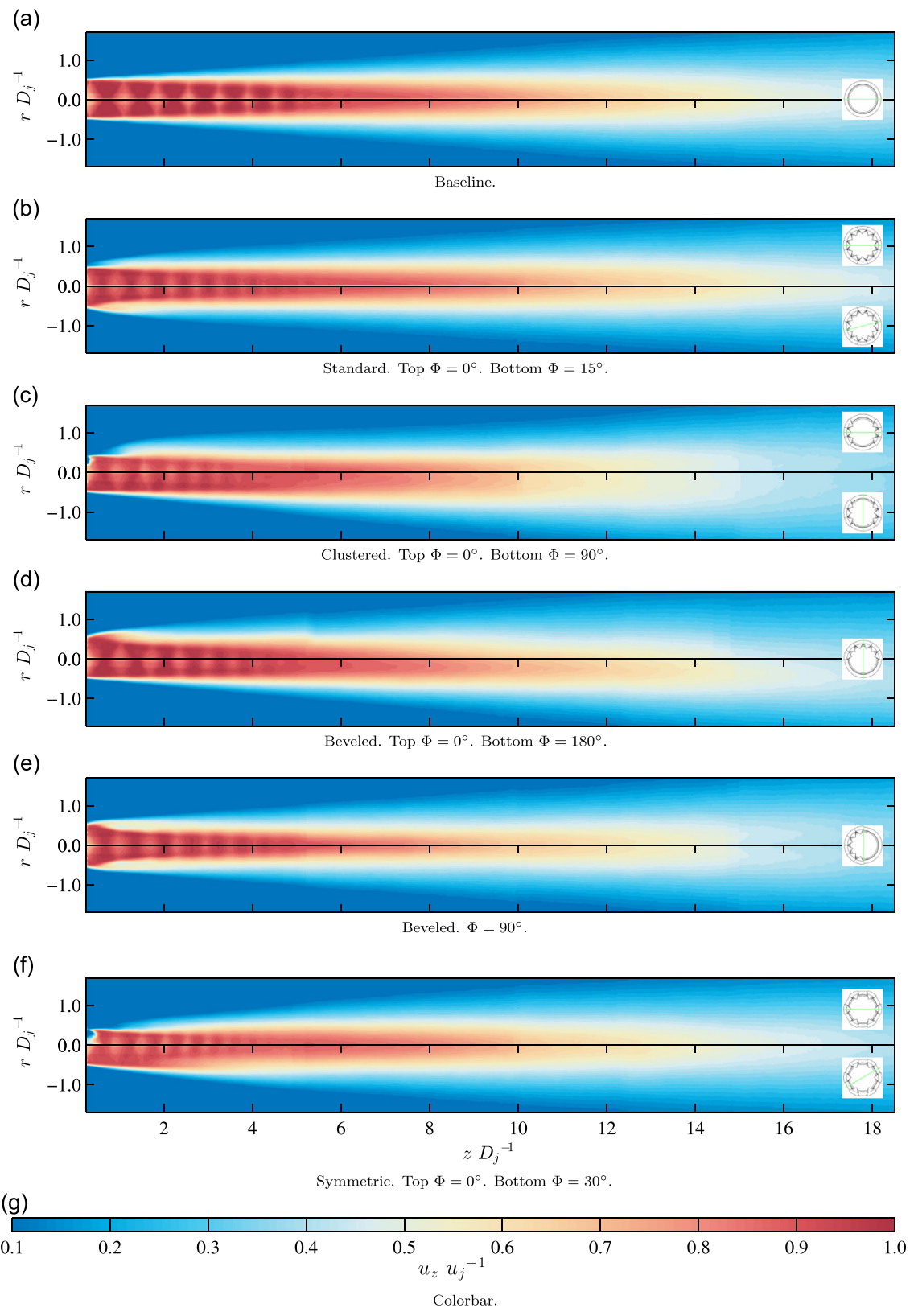

Fig. 17. Streamwise planes of mean axial velocity. $M_{j}=1.22$. 
spacing was roughly axisymmetric in both the tip and valley planes, indicating limited azimuthal effect on the high speed jet core. The clustered configuration's increased spreading, which resulting in the elliptic cross section, was easily seen in the $\Phi=90^{\circ}$ plane. Due the presence of the chevron tip in the $\Phi=0^{\circ}$ plane, an expulsion of fluid from the jet core was not measured until roughly a diameter downstream, which corresponded to the position where the jetlets begin to merge, as shown in Fig. 16d. Additionally, an asymmetric shock structure was apparent near the nozzle in the first three shock cells. Finally, due to the drastic increase in spreading, the clustered configuration appeared to have drastically reduced the potential core length, a feature which will be quantitatively discussed below. Due to the complexity of the beveled configuration, three streamwise planes were measured at $\Phi=0^{\circ}, 90^{\circ}$, and $180^{\circ}$. Since the $\Phi=0^{\circ}$ and $180^{\circ}$ planes are co-planer, they were combined in Fig. 17d. As expected from the above cross-sections, a large amount of fluid was expelled radially outward due to the presence of the chevrons, as seen in the lower half of Fig. 17d. The initial development of the portion of
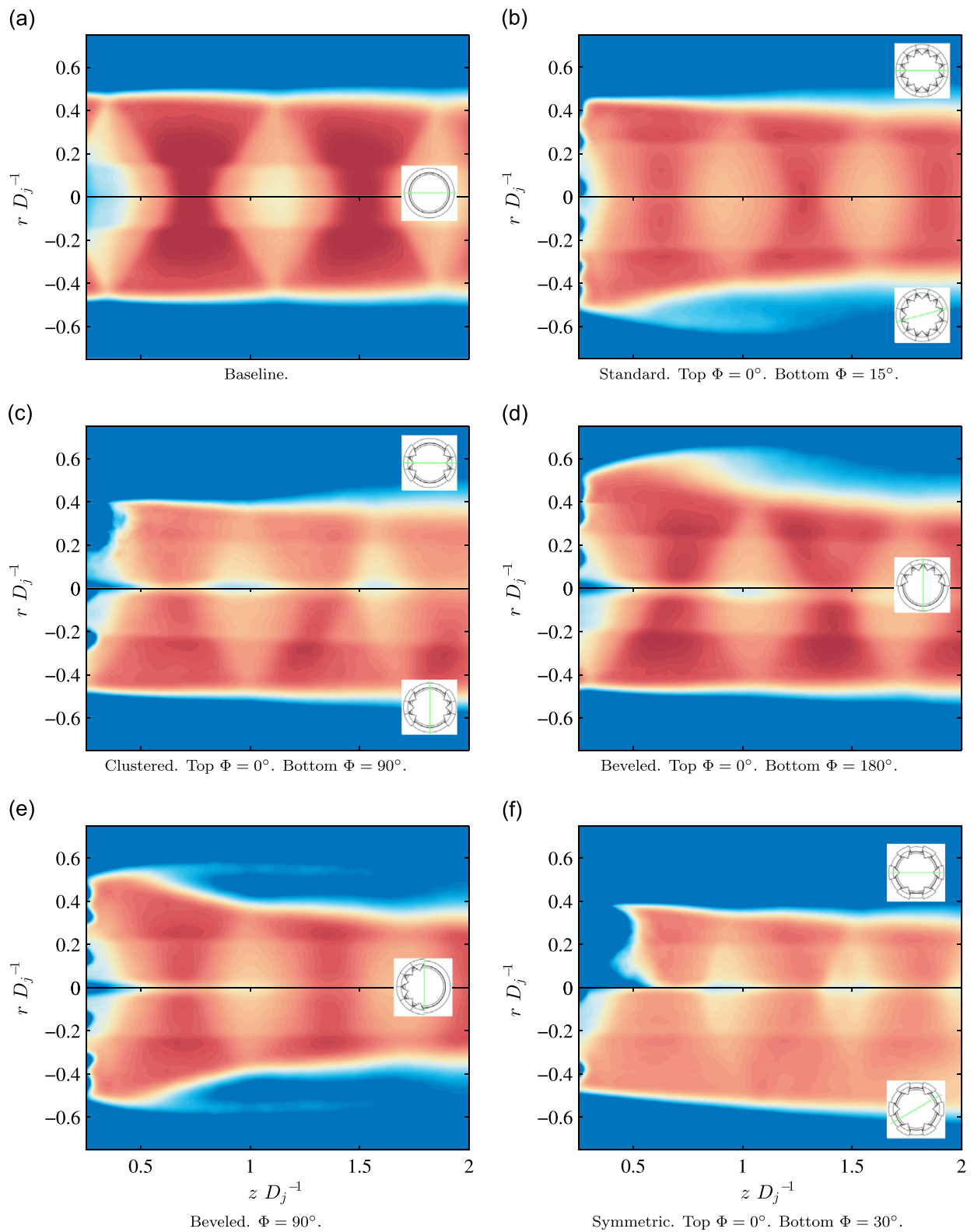

(g)

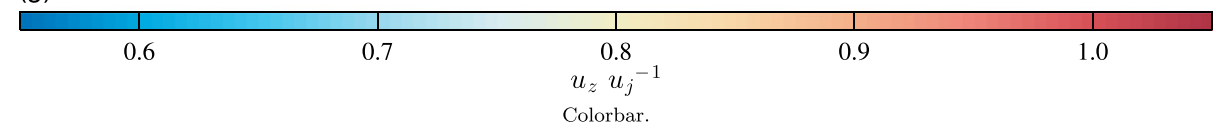

Fig. 18. Enlarged near nozzle view of streamwise planes of mean axial velocity. $M_{j}=1.22$. 
the jet without chevrons was similar to the baseline configuration, but as the jet propagated downstream it was deflected off of the centerline in the direction opposite of the chevrons. Beveled jets are known to deflect from the centerline [19,20], but the deflection of overexpanded jets is typically in the direction of the long side of the jet. This is primarily due to inward turning of the flow at the lip of the shorter side of the bevel. As there was pressure relief between the chevrons it is thought that the asymmetric inward turning is limited in the present case. Consequently the jet deflection is thought to result simply from the penetration of the chevrons into the flow. An estimate of the deflection angle was calculated using several downstream velocity profiles, resulting in a value of roughly $1.7^{\circ}$. A drastic reduction in the radial extent of the jet appeared to occur in the $\Phi=90^{\circ}$ orientation, but as the measurement plane was oriented along the geometric centerline, the

(a)

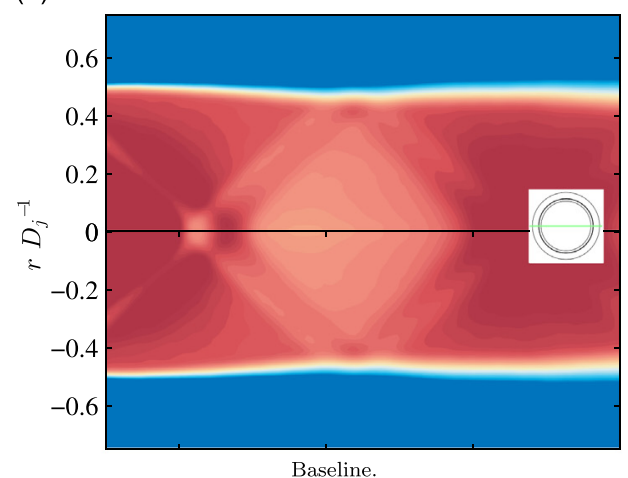

(c)

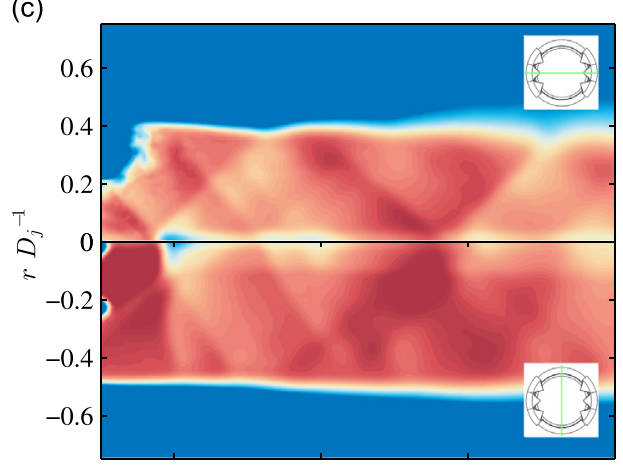

Clustered. Top $\Phi=0^{\circ}$. Bottom $\Phi=90^{\circ}$

(e)

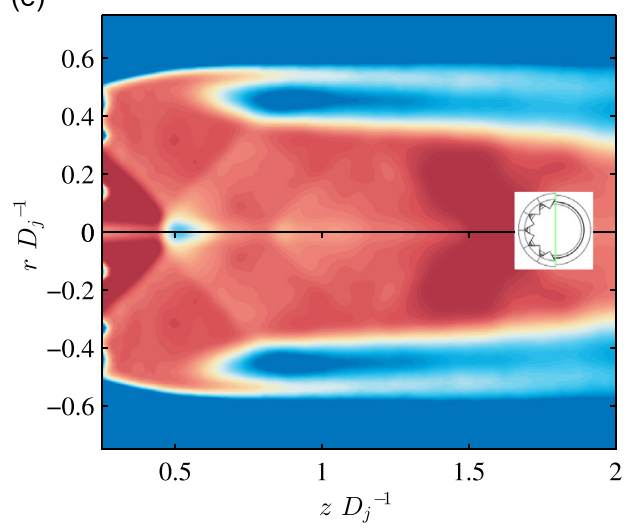

Beveled. $\Phi=90^{\circ}$

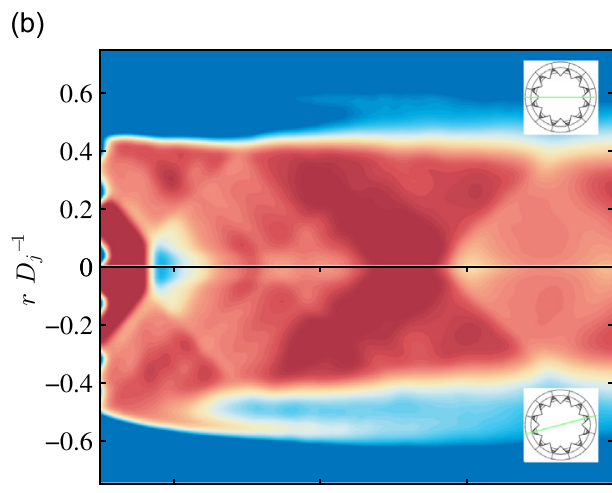

Standard. Top $\Phi=0^{\circ}$. Bottom $\Phi=15^{\circ}$

(d)

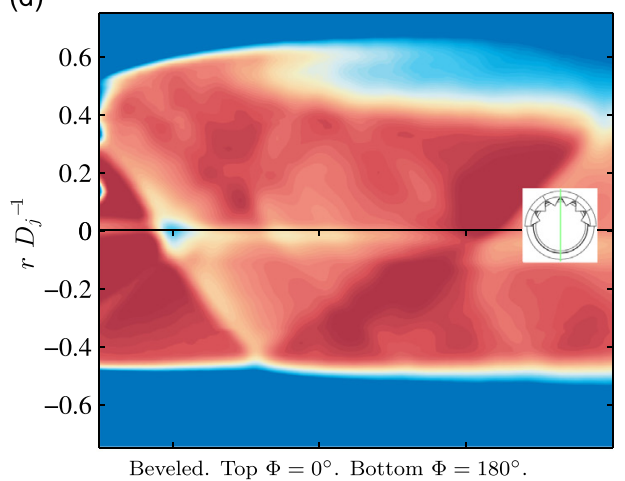

(f)

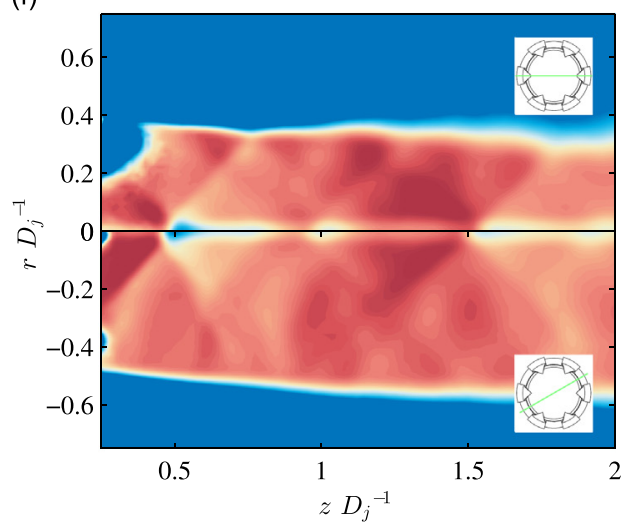

Symmetric. Top $\Phi=0^{\circ}$. Bottom $\Phi=30^{\circ}$.

(g)

$$
0.6
$$$$
0.7
$$

$$
u_{z}^{0.8} u_{j}^{-1}
$$

Fig. 19. Enlarged near nozzle view of streamwise planes of mean axial velocity. $M_{j}=1.50$. 
deflection of the jet described above exaggerates this effect. Additionally, side lobes were present in this orientation, particularly evident in Fig. 18e and Fig. 19e, which resulted from the measurement plane intersecting the non-radially propagating jetlets introduced by the chevrons located on the azimuthal extremes of the group of chevrons. Finally, the symmetric configuration caused a large expulsion of fluid in the region between the chevrons, which is easily seen in the lower half of the figures. This feature persisted much further downstream compared to the other investigated configurations, with a region of velocity of similar magnitude to the core jet extending roughly to three diameters downstream. This allowed the shock structure to exist within the crenelations of the jet, resulting in a three dimensional shock structure as indicated by the shock diamonds in Fig. $17 \mathrm{f}$. In all cases the shock cell spacing was reduced by the application of chevrons, a feature which will be disused further below.

Next, the enlarged near nozzle regions of the ideally expanded condition are presented in a similar manner to the overexpanded condition above. Increasing the jet Mach number drastically modified the baseline configuration, with limited-to-no Mach disk present and the typical double shock diamond structure easily identifiable. The shock structure of the standard configuration, on the other hand, was highly complex and included a strong centerline Mach disk. Following
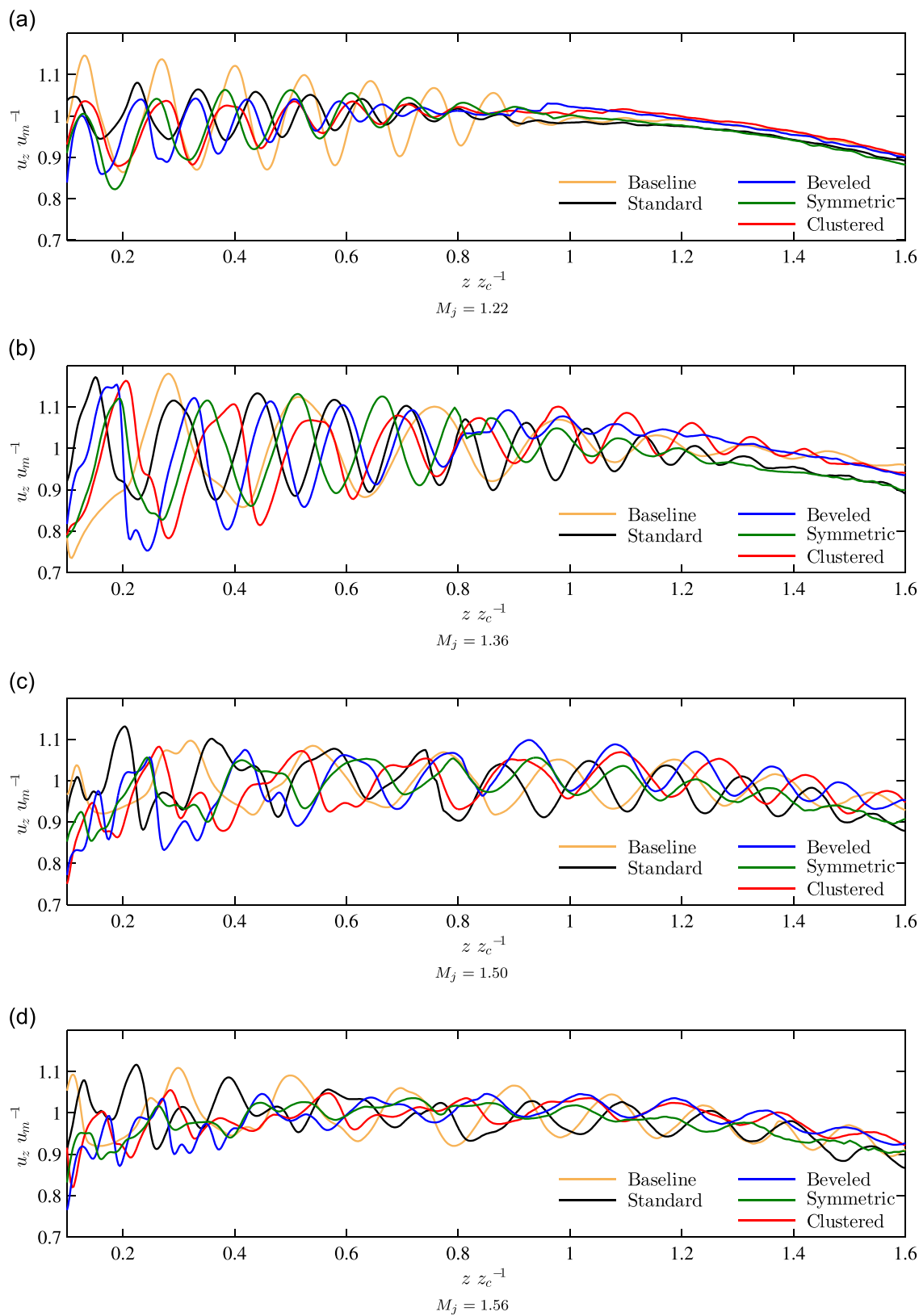

Fig. 20. Centerline profiles of axial velocity normalized by the mean centerline velocity within the potential core. The axial coordinate is normalized by the potential core length. 
the overexpanded results, the standard configurations shock structure was roughly axisymmetric, most likely due to the rotational symmetry of the chevron arrangement. Additionally, a large expulsion of high velocity fluid is also generated between the chevrons $\left(\Phi=15^{\circ}\right)$. Comparison to the baseline flow field indicated an overall reduction in shock spacing as well as the radial extent of high velocity fluid, most likely due to increased mixing. Similarly, the clustered configuration introduced a complex shock structure and reduced the shock cell spacing. Unlike the standard configuration, the clustered jet's shock cell structure was highly azimuthal due to the induced elliptic cross-section of the developing jet. The differing spread rates between the $\Phi=0^{\circ}$ and $90^{\circ}$ planes is visible in aft portion of Fig. 19c; this becomes more pronounced further downstream, resulting in the elliptic cross-section discussed above. The beveled configuration also drastically modified the shock cell structure, with the presence of the chevrons introducing a much larger magnitude system than the portion of the nozzle without modification. The core of the jet was again deflected away from the centerline in the direction opposite of the chevrons. The deflection angle was determined to be roughly $1.5^{\circ}$, following the manner discussed above. The large sidelobes present in the $\Phi=0^{\circ}$ orientation were again due to the location of the measurement plane and the non-radially propagating jetlets, as described above. Comparison to the standard configuration indicated a larger expulsion of fluid was achieved in the $\Phi=0^{\circ}$ by the beveled configuration and that the shock reduction was not as large, though comparison is somewhat difficult due to the canted centerline. Finally, similar to the other configurations, the symmetric configuration resulted in a complicated shock structure compared to the baseline flow field. This was primarily due to the expulsion of fluid between the chevrons being supersonic and coherent enough to sustain shocks, as seen in Fig. 19f. Consequently, the shock reflection locations were axially disparate, resulting in an asymmetric shock/expansion system. The high speed expulsion persisted down to roughly four diameters downstream (not shown) after which the jet takes on the typical shock/ expansion system.

In an effort to quantitatively determine modifications to the flow field by the investigated configurations, axial velocity profiles were extracted from the measured flow fields. The first of which was the centerline velocity profiles (shown below in Fig. 20) for use in determining the potential core length of each of the configurations. Due to the deflection of the beveled configuration from the geometric centerline, a canted centerline following the flow features was used. In Fig. 20, the velocities were normalized by the mean axial velocity within the potential core and the axial coordinate was normalized by the potential core length, the determination of which will be outlined below. As seen, qualitative comparison of the centerline profiles is difficult due to the complexity of the shock cell/Mach disk system. The single shock diamond structure present for all configurations in the overexpanded regime is apparent from the sinusoidal profiles in Fig. 20a and b. In the ideally and under expanded regimes, the double shock cell structure extended through a majority of the potential core, which collapsed into a single shock structure that persisted past the end of the theoretical potential core termination. The amplitude and frequency of velocity fluctuation at the most overexpanded condition indicates a reduction in shock strength and spacing by the investigated configurations, but at the higher jet Mach numbers the complexity and differences in the flow fields makes qualitative judgments impractical. Consequently, quantitative analysis of the velocity profiles was performed to extract the pertinent parameters.

Determination of the potential core length was performed using a slightly modified version of the centerline velocity profile developed by Lau et al. [59] (shown below in Eq. (3)) following the methodology of Heeb et al. [24]:

$$
u_{z}(z) / u_{m}=\left\{\begin{array}{ll}
1 & z \leq z_{c} \\
1-\exp \left(\frac{\alpha}{1-z / z_{c}}\right) & z>z_{c}
\end{array}: \quad \alpha=1.5, u_{m}=\overline{u_{z}\left(z \in\left[0, z_{c}\right]\right)}\right.
$$

The modification of the original profile consisted of inclusion of the average centerline velocity modification term, $u_{m}$, which accounted for the non-constant velocity within the potential core due to the presence of the shock/expansion system. This result was least squares fit to each of the extracted centerline velocity profiles, which easily affords the potential core length through simple back calculation. The minimum coefficient of determination $\left(R^{2}\right)$ of all of the data fits was 0.95 , indicating an excellent agreement between the centerline velocity and Lau et al.'s [59] profile and an accurate determination of the potential core length.

Fig. 21 shows the result of completing this routine for all available streamwise data.Additionally, the prediction of Lau et al. [59] shown below in Eq. (4) was included for comparison purposes:

$$
\frac{z_{c}\left(M_{j}\right)}{D_{j}}=4.2+1.1 M_{j}^{2}
$$

The baseline potential core length at the lowest and highest measured jet Mach numbers were reasonably captured, though the $M_{j}=1.36$ and $M_{j}=1.50$ conditions significantly deviated from the profile. As noted above, this is associated with large amplitude jet column undulation caused by screech, originally documented by Glass [39] and more recently Bridges and Wernet [40]. Consequently, direct comparison between the baseline jet's potential core length and the investigated chevron cases was difficult as screech amplitude was drastically reduced by all of the chevron configurations as shown above in Fig. 7. Comparison to the standard configuration allowed an assessment of the effect of arrangement on the potential core length independent of screech. As expected from the presented contour plots, the clustered configuration optimally reduced the potential core length compared to the other chevron configurations across the entire operating range, which follows previously presented elliptic jet results $[60,15]$. Interestingly, when considering only the chevron configurations, potential 


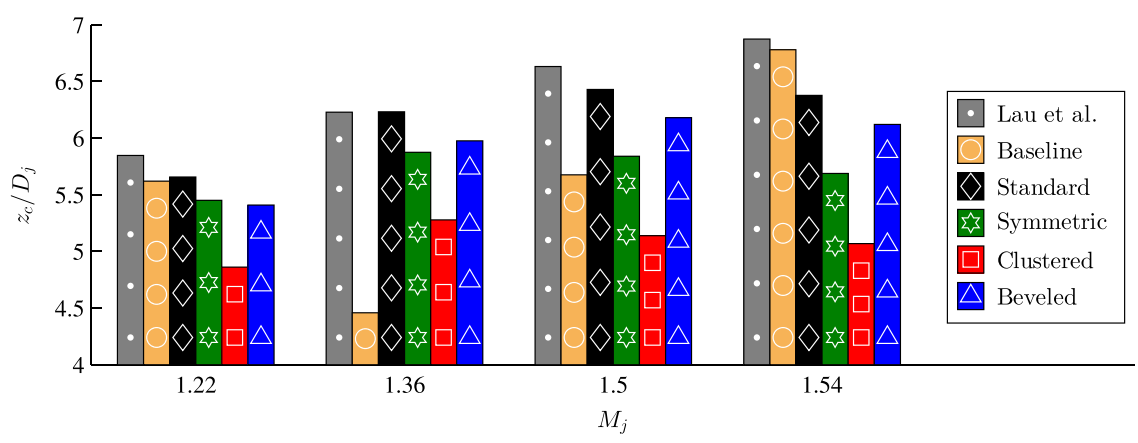

Fig. 21. Potential core length comparison along with the prediction of Lau et al. [59].

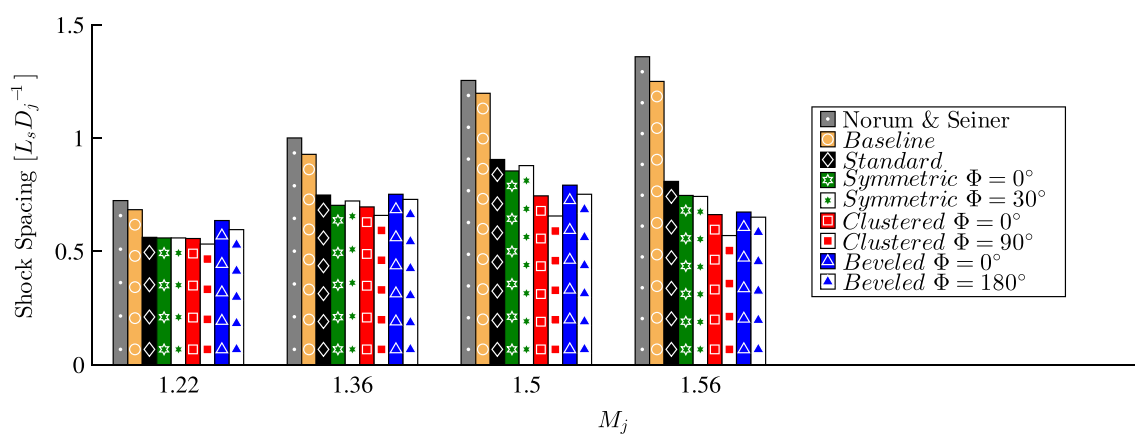

Fig. 22. Comparison of shock cell spacing with the relation of Norum and Seiner [61].

core length modification is entirely configuration dependent. The standard configuration possessed the longest potential core length followed by the beveled, then symmetric, and finally clustered configuration. This indicates azimuthal arrangement cannot be simplified down to a total chevron projected area metric, at least in-terms of predicting the change in potential core length.

Following the methodology outlined in Heeb et al. [44], analysis of the shock cell structure was also performed using extracted axial velocity profiles. To effectively determine azimuthal asymmetry in the shock cell structure, velocity profiles were extracted at a radial distance 0.15 diameters away from the centerline. This location was chosen as a compromise between moving radially away from the jet centerline to effectively identify azimuthal asymmetry and the axial length of the profile before the extraction line intersected with the jet shear layer. The beveled configuration's $\Psi=90^{\circ}$ plane was excluded from this analysis due to the misalignment of the geometric and jet centerlines. First, the mean shock spacing was determined by averaging the shock spacing determined by consecutively subtracting the axial locations of the profile's peak velocity. Only the first six shock cells were used to eliminate the effect of screech on the calculated parameter, following the results of André et al. [43]. Fig. 22 presents the results of this effort along with Norum and Seiner's [61] empirical modification of the Prandtl-Pack [62] vortex-sheet model for CD nozzles, given below in the following equation:

$$
\frac{L_{s}}{D_{j}}=a\left(M_{j}^{2}-1\right)^{b / 2} ; \quad a=1.1 \text { and } b=1.17
$$

The relation of Norum and Seiner [61] reasonably followed the baseline data with only a slight over prediction throughout the operating range. This over prediction is thought to be a result of the use of a non-centerline profile, which excludes the more normal portion of the shock structure for the calculations herein. Investigation of the chevron configurations indicated that regardless of configuration, orientation, or operating condition, the shock cell spacing was reduced by the application of chevrons. The symmetric configuration shows limited azimuthal variance in the shock cell spacing, which confirmed the roughly constant acoustic BSAN peak frequency presented above in Fig. 9. Additionally, the shock spacing was reduced below the level of the standard configuration for all operating conditions independent of azimuthal orientation. The shock spacing of the clustered configuration did show some azimuthal variation, with the $\Phi=0^{\circ}$ possessing a longer spacing than the $\Phi=90^{\circ}$ throughout the operating range. This also validates the BSAN amplitude results presented above where the $\Phi=90^{\circ}$ orientation had a higher peak Strouhal number across all operation conditions following the inverse relation between shock spacing and peak frequency. As with the symmetric configuration, the shock spacing was further reduced as compared to the standard configuration, especially at the higher operating conditions. Finally, the beveled configuration also presented azimuthal variation of shock cell spacing with the $\Phi=180^{\circ}$ achieving a shorter spacing across the operating range. The unilateral reductions in comparison to the standard configuration were not achieved by the beveled configuration, with the spacing in the overexpanded regime exceeding the standard configuration. Back calculation 
of the BSAN peak frequency assuming the convective velocity is 70 percent of the fully expanded jet velocity [63] (Eq. (2)) indicated that the relation between the configuration and the peak frequency increase was captured using this analysis method. Discrepancies in absolute levels exist, particularly at the overexpanded conditions. This is possibly due to the larger jet spread at the overexpanded conditions [64], which introduced an increasingly non-orthogonal length measurement of the shock spacing when using a non-centerline profile.

In addition to the mean shock cell spacing, the average shock strength was also calculated using the velocity profiles extracted at 0.15 diameters off of the centerline. Shock strength is defined here following the manner of Heeb et al. [24] as the difference between the upstream velocity maximum and downstream minimum velocity divided by the fully expanded velocity $\left(u_{1}-u_{2}\right) u_{j}^{-1}$. Following the reasoning of Tam [50], which indicated that the upstream Mach disk was uncorrelated with shock noise levels, the most upstream shock strength was excluded from the average calculation. Additionally, the number of shock cells used for the strength calculation was limited to six following the results of André et al. [43] as discussed above. A slightly modified shock strength characterization parameter of Tam [50] was developed for comparison purposes. The modification to Tam's shock strength parameter is the inclusion of an additive constant to account for the

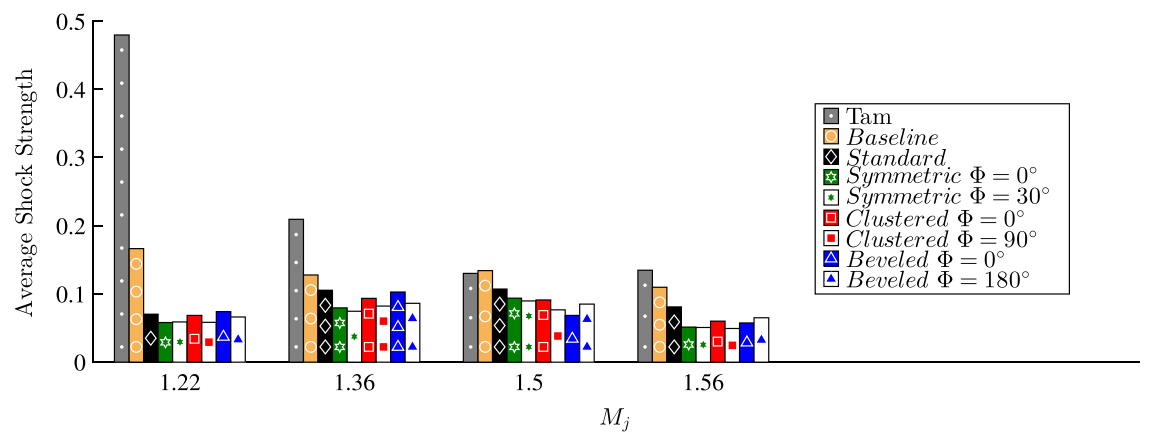

Fig. 23. Average shock strength as a function of fully expanded jet Mach number. Tam's [50] strength parameter provided for comparison.

(a)

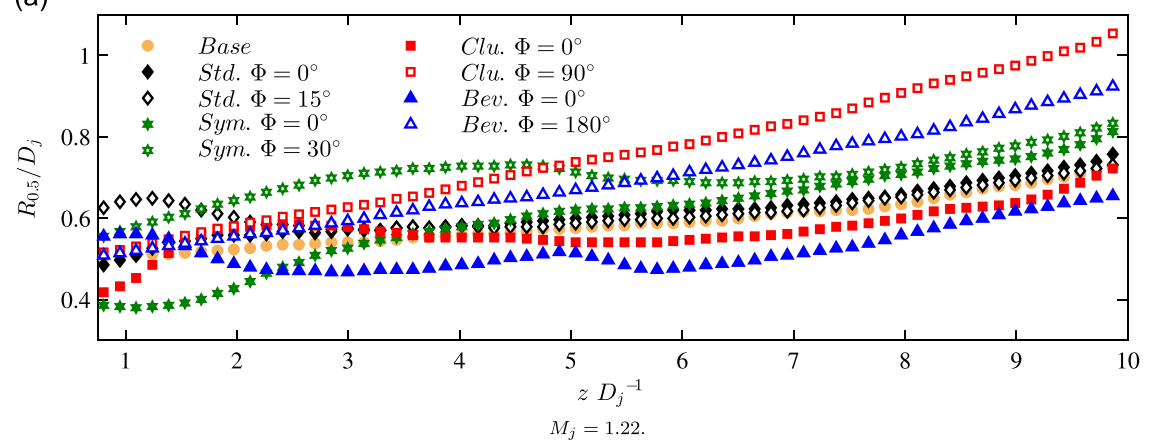

(b)

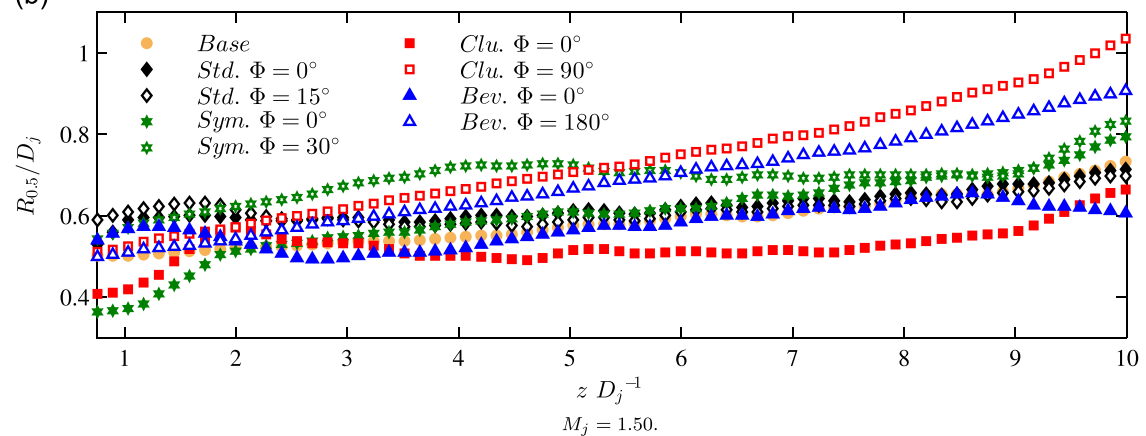

Fig. 24. Normalized jet half-width development. 
occurrence of shock cells in the jet at the design condition:

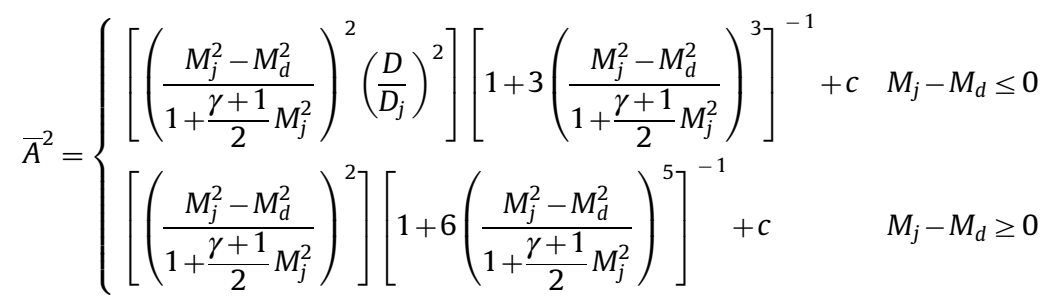

This constant was determined to be equal to 0.13 , and as expected reasonably aligns the prediction with the empirically measured baseline shock strength. Tam's original development of Eq. (6) was for moderately imperfectly expanded jets from smooth nozzles and is consequently only valid for the jet Mach number range which just encompasses the maximum BSAN in the over and under expanded regimes. The results of Munday et al. [25] indicate that this valid Mach number range for the nozzle under investigation here is $1.36 \leq M_{j} \geq 1.80$. Therefore, the shock strength at the $M_{j}=1.22$ condition was expected to be grossly over predicted by Eq. (6), and some over prediction was possible for the $M_{j}=1.36$ as it is at the valid range cutoff.

The results of the average shock strength calculation along with Eq. (6) are shown in Fig. 23. As expected from the above discussion, the baseline jet's average shock strength at the two overexpanded conditions were over predicted by the relation of Tam [50]. Comparison of the chevron configurations to the baseline jet indicated a reduction in shock strength irrespective of operating condition, configuration, or azimuthal arrangement. The largest amplitude reductions compared to the baseline configuration were achieved at the extreme ends of the investigated operating range, which follows the acoustics results. The symmetric configuration shows limited azimuthal variation in the shock spacing which corroborates the BSAN amplitude discussion above. Average reduction compared to the standard configuration is just under 24 percent. The clustered configuration possessed some azimuthal variation, with the $\Phi=0^{\circ}$ orientation having a 15 percent higher strength on average. This follows that the BSAN amplitude results which indicated the peak BSAN amplitude at $\Phi=0^{\circ}$ was louder than the $\Phi=90^{\circ}$ plane. Due to the under performance of the $\Phi=0^{\circ}$ orientation, on an azimuthally averaged basis the clustered configuration achieved a lower reduction than symmetric configuration (20 percent relative to the standard
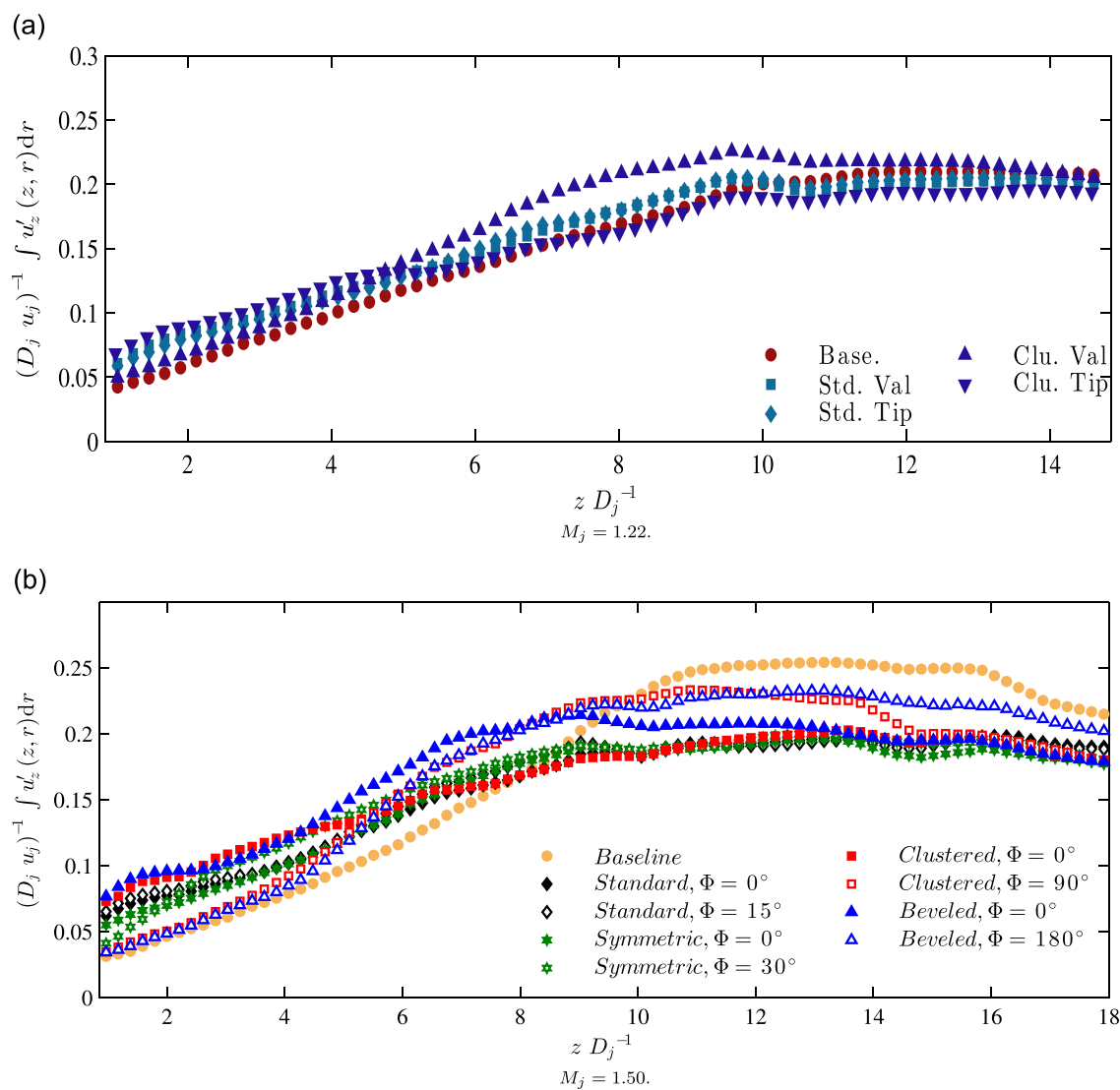

Fig. 25. Normalized integrated axial turbulence development. 
configuration). Finally, the beveled configuration also presented azimuthal variation in average shock strength, which switches optimum orientation in the over vs. ideally/under expanded regimes, which was mirrored in the BSAN amplitude results presented above. This was a result of the change in the shock cell structure from roughly diamond shaped in the overexpanded regime into a system comprised primarily of a single strong oblique shock/expansion train. In general the beveled configuration was out performed by the symmetric and clustered configurations, except at the ideally expanded condition where the optimum shock strength (and peak BSAN amplitude) reduction was achieved by the beveled configuration.

In addition to the above quantities, the streamwise velocity data was used to investigate the spread rate characteristics of the different configurations. Jet half-width was selected from the metrics presented in Schadow et al. [64] to emphasize the spread characteristics as it best differentiated the near nozzle and downstream flow fields examined herein. To account for non-constant centerline velocity within the potential core, the jet half-width was defined within this work as the radial location where the local velocity magnitude normalized by the local maximum velocity is equal to $1 / 2$. The over and ideally expanded jet half-width results are located in Fig. 24 for all of the investigated configurations excluding the beveled configuration's $\Phi=90^{\circ}$ orientation due to the misalignment of the measurement plane and the flow centerline. Due to the large amount of axial data, only every tenth point was included to ease visualization. The baseline and chevron results followed similar trends to the half-width presented by Schadow et al. [64], but due to the mismatch in jet Mach number, the rates were not directly comparable as rate decreases with Mach number [65]. In the very near nozzle region $\left(z D_{j}^{-1}<2\right)$, the standard configuration $\left(\Phi=15^{\circ}\right)$ produced the largest increase in jet half-width, compared to the baseline configuration, though the differences were larger in the overexpanded regime. By roughly 5 diameters downstream, both of the standard configuration's investigated orientations returned to the baseline half-width. The symmetric configuration possessed the largest initial decrease in jet half-width due to the presence of the chevron tip $\left(\Phi=0^{\circ}\right)$. As shown by the cross-sectional velocity contours above, the symmetric configuration's increased chevron azimuthal spacing did not result in a typical jetlet structure, but instead a lobed structure that persisted further downstream. This is evident in the $2<z D_{j}^{-1}<5$ range where the symmetric configuration had the largest jet half-width. The azimuthal variation in the symmetric configuration's halfwidth collapses by roughly 7 diameters downstream. As the measurement planes used to investigated the clustered configuration contained either a chevron tip $\Phi=90^{\circ}$ or an unmodified portion of the nozzle, an increase in the measured near nozzle jet half-width similar to the other configurations was not captured. Aft of roughly 2 diameters, the modification of the jet cross section to an ellipse became apparent as the jet half-width of the $\Phi=0^{\circ}$ and $\Phi=90^{\circ}$ planes diverged. As expected the resulting elliptic flow field's major axis exceeded all other conflagration's half-width for both the overexpanded and ideally expanded conditions downstream of roughly 5 diameters. The beveled configuration possessed an initial increase in jet half-width in the $\Phi=0^{\circ}$ plane due to the expulsion of fluid between the chevrons, while the $\Phi=180^{\circ}$ orientation initially followed the baseline profile, as no modification was made the nozzle in that azimuthal plane. Due to

(a)

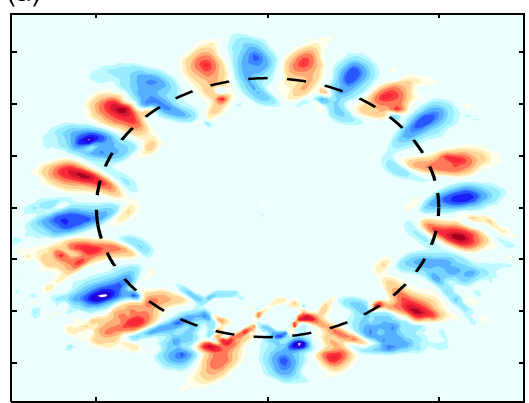

Standard. (b)

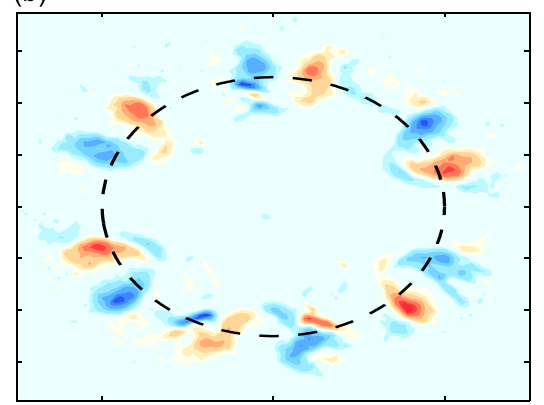

Symmetric (c)

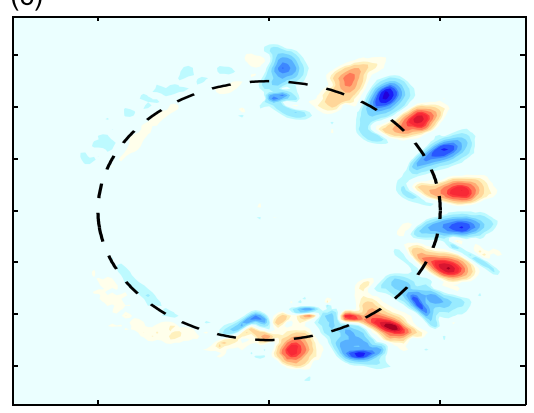

Beveled. (d)

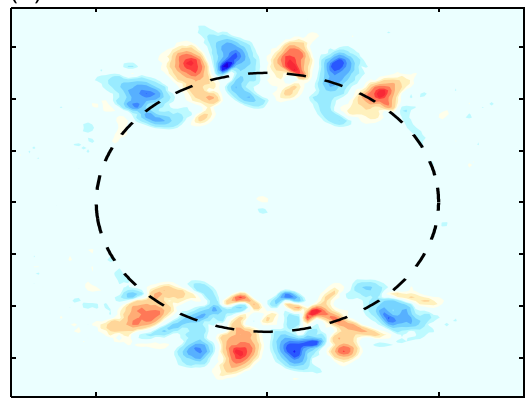

Clustered. (e)

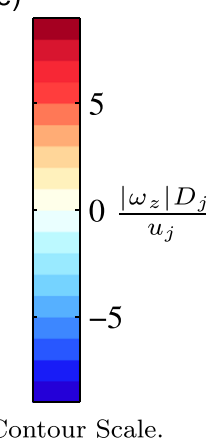

Fig. 26. Streamwise vorticity at $z / D_{d}=0.5, M_{j}=1.22$. 
the jet deflection off of the centerline away from the chevrons, the $\Phi=180^{\circ}$ jet half-width increased downstream to values above the baseline and all other configurations excluding the clustered configuration in the $\Phi=90^{\circ}$ orientation. Conversely, the $\Phi=0^{\circ}$ orientation's downstream half-width is smaller than most of the other configurations due to the misalignment between the jet and geometric centerlines.

(a)

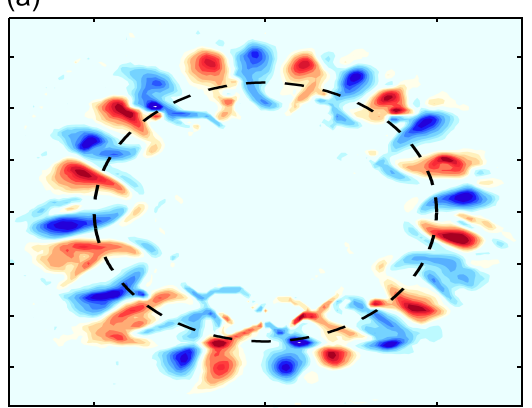

Standard. (b)

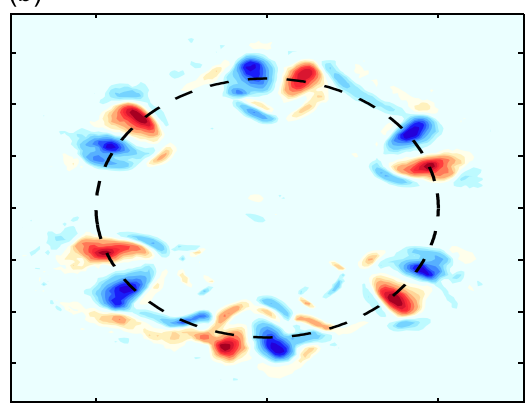

Symmetric. (c)

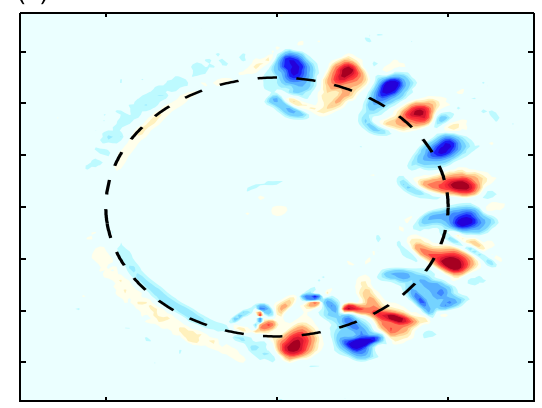

Beveled. (d)

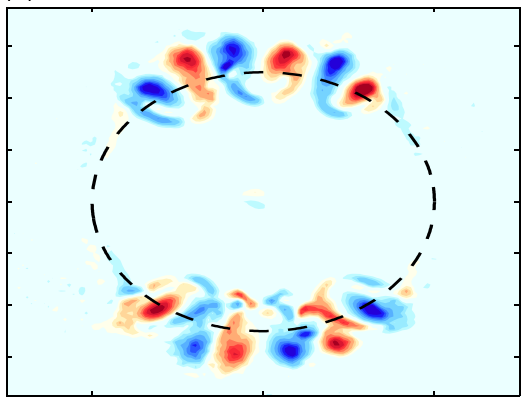

Clustered. (e)

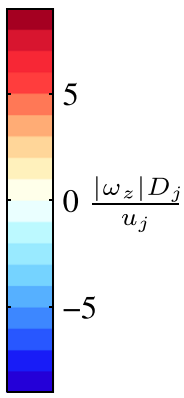

Contour Scale.

Fig. 27. Streamwise vorticity at $z / D_{d}=0.5, M_{j}=1.50$.

(a)

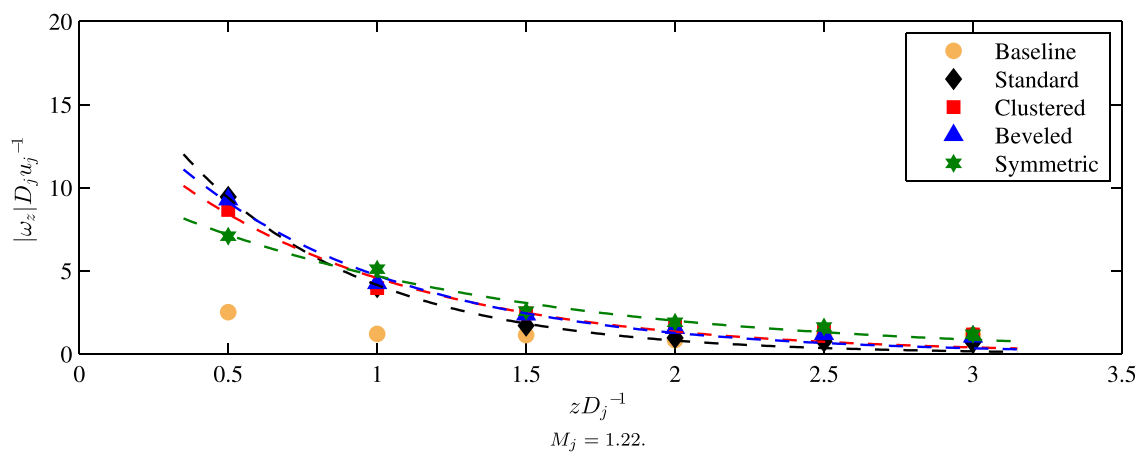

(b)

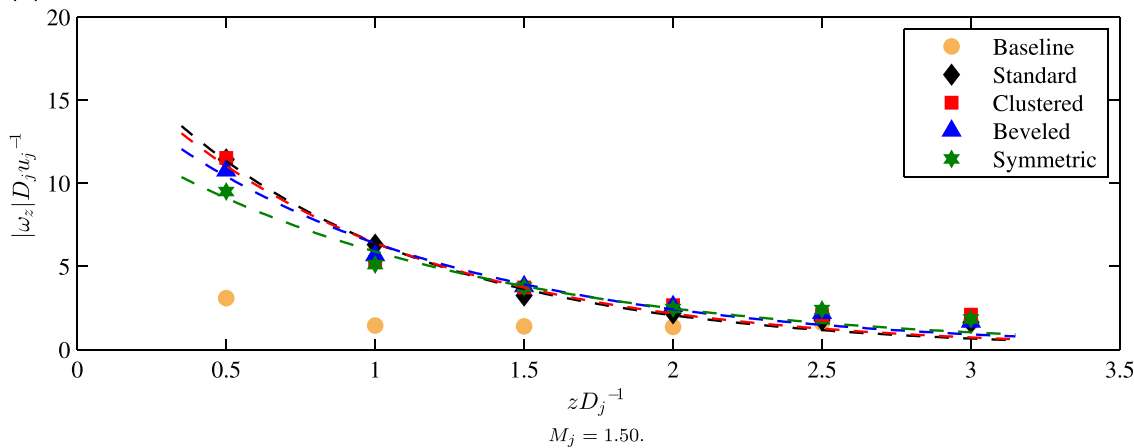

Fig. 28. Variation of maximum streamwise vorticity with downstream position. 
Finally, integration of the fluctuating axial velocity measurements was completed following the reasoning of Bridges et al. [5] as it relates to mixing noise source strength. The resulting quantity, normalized by the fully expanded jet velocity and diameter, is presented in Fig. 25. Similar to the half-width results above, only every twelfth data point is plotted for clarity. The baseline configuration exhibited a roughly linear increase in integrated turbulence until roughly 10 diameters downstream, where the levels plateau for both the over and ideally expanded conditions. At the overexpanded condition, the very near nozzle results indicated increased integrated turbulence by all investigated chevron configurations, particularly in the measurement planes containing jetlets as discussed above. The integrated turbulence was increased universally upstream of 10 diameters. Downstream of that location, reductions up to or below the level of the baseline jet were observed for all of the configurations excluding the clustered $\Phi=90^{\circ}$ and beveled $\Phi=180^{\circ}$ orientations. These configurations exceed the baseline jet's values due to measurement aligned with the major axis of the elliptic cross section and canted jet centerline, respectively. Furthermore, this confirms the discussion regarding the requirement of localized streamwise vorticity for inhibited large scale structure growth and consequent LSS mixing noise, as shown by the comparison between the two clustered and/or beveled orientations. The symmetric configuration resulted in the lowest integrated downstream turbulence, indicating an optimal inhibition of large scale growth [5], which confirms the peak LSS amplitude results presented in Fig. 11. The presence of relatively large amplitude screech at the design condition is known to increase the levels of downstream turbulence as compared to the expected levels from a non-screeching axisymmetric jet [40]. Consequently, comparison to the baseline results at the design condition is difficult, as the chevron configurations all but eliminated screech. A near nozzle increase in integrated turbulence was again initiated by all of the investigated chevron configurations due to the introduction of small scale structures. Similarly, the clustered $\Phi=90^{\circ}$ and beveled $\Phi=180^{\circ}$ resulted in the highest downstream turbulence, following the discussion above. The symmetric and beveled $\Phi=0^{\circ}$ ordinations achieved comparable downstream turbulence levels, again confirming the LSS reduction results presented above.

The flow field modifications discussed above were a result of the introduction of counter rotating vortex pairs into the jet by the chevron configurations. Figs. 26 and 27 depict the near nozzle streamwise vorticity normalized by the fully expanded jet velocity and diameter for the overexpanded and ideally expanded conditions, respectively. For reference, the nozzle lip line was superimposed onto the figures to aid in identifying expulsion and penetration of the vortices. In overexpanded operation the standard configuration produced the highest vorticity magnitude followed by the beveled, clustered, and finally the symmetric configuration. This trend indicates that an uninterrupted chain of chevrons is beneficial in terms of vorticity magnitude. Proximity of the vortices limited the azimuthal growth, leading to elongated vortex shape and increased interaction. It is interesting to note that maximum near nozzle vorticity did not correlate to increased downstream cross section modification, as seen by comparing standard and symmetric configuration jet cross sections (Fig. 15). At the ideally expanded condition, the difference between peak vorticity magnitude was reduced, though the symmetric configuration still achieved the lowest values. Increases in the radial extent of the vortices resulted for all but the symmetric

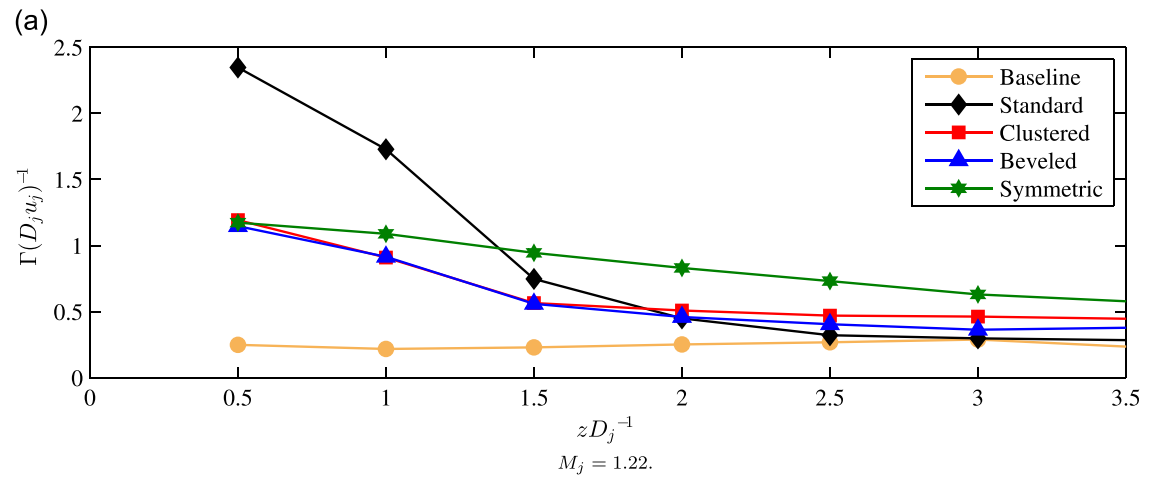

(b)

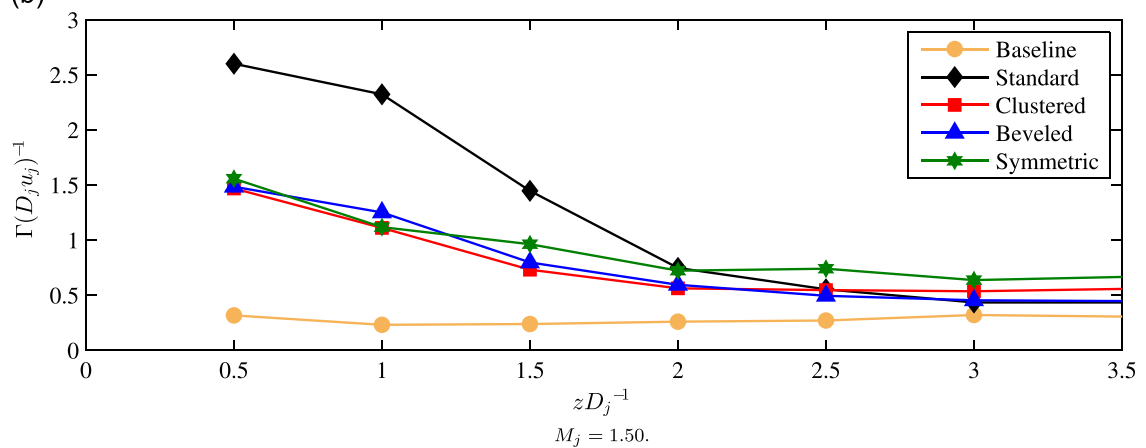

Fig. 29. Variation of $\Gamma$ with downstream position. 
configuration, most likely due to a lack of proximity. Correspondingly, there was a reduction in the vortex coherence with an increase in vortex radial extent. Additionally, secondary vortex structures developed between adjacent main pairs at the radial extent of the vorticity field. The direction of rotation of these secondary structures was opposite compared to the primary pair. Burak et al. [66] presented similar vorticity features in a computational study of chevrons.

In an effort to quantitatively assess the impact of chevron arrangement on the induced vorticity field, the maximum vorticity was tabulated at each available axial location, as presented in Fig. 28, for the over and ideally expanded conditions. Following the reasoning of Alkislar et al. [57], an exponential function was fit to each data set to illustrate the peak decay rate. All configurations showed similar maximum vorticity values and decay rates, though the symmetric configuration induced a lower near nozzle maximum vorticity than the other configurations. Following the discussion above, this is thought to result from the reduced proximity of the induced vortex pairs which limited vortex diffusion. Downstream, the symmetric configuration's maximum vorticity was larger than the other configurations, possibly indicating initial vortex interaction results in reduced persistence. Though these differences are observable, the primary result was that chevron arrangement has limited effect on the maximum vorticity in comparison to chevron geometry, as presented by Heeb et al. [24].

Following the reasoning of Heeb et al. [24], a metric that included both magnitude and spatial extent was deemed to more accurately assess the effect of chevron arrangement on streamwise vorticity. This metric involves integrating the modulus of streamwise vorticity at the available jet cross section as defined in the following equation:

$$
\Gamma=\int_{c s}\left|\omega_{z}\right| \mathrm{d} c s
$$

Fig. 29 displays the resulting decay profiles of the $\Gamma$ quantity nondimensionalized by the fully expanded jet velocity and diameter. Unlike the maximum vorticity profiles, the results were not found to decay exponentially. As expected from the near nozzle vorticity contours presented in Figs. 26 and 27, the standard configuration resulted in a higher near nozzle $I$ value than the other configurations. The decay rate of the standard configuration is higher than the other configurations, leading to similar values by two diameters downstream. The clustered and beveled configurations resulted in similar values in both the overexpanded and ideally expanded regimes. The symmetric configuration displayed the lowest decay rate of the investigated configurations, resulting in higher downstream values. This further illustrates the improved persistence of the symmetric configuration due to reduced vortex interaction and coincides with the LSS peak amplitude and the downstream integrated turbulence reductions presented above.

\section{Conclusions}

A study of the effects of chevron spacing and asymmetric distribution on a supersonic jet's flow and acoustic field was performed in an effort to illuminate the potential benefits of optimizing more than just individual chevron geometry. To this end, three differing arrangements were created from a set of six individual chevrons with fixed geometry: beveled (all six chevrons grouped to one side of the nozzle), clustered (two groups of three chevrons assembled towards opposite sides of the nozzle), and symmetric (six chevrons equally spaced around the nozzle perimeter). These were compared to a standard chevron arrangement consisting of 12 chevrons encompassing the entirety of the nozzle perimeter, as well as an unmodified baseline nozzle. Investigation of the overexpanded, ideally expanded, and underexpanded regimes was performed using streamwise and cross stream PIV flow field measurements along with far-field acoustic measurements. This allowed correlation between axial and radially aligned jet features such as shock spacing and streamwise vorticity and modifications of the primary noise sources.

The most identifiable flow field modification achieved by the investigated configurations was the drastic change in cross sectional shape. Near nozzle jetlets were introduced by all of the configurations except the symmetric configuration, which was a result of the increased chevron spacing reducing vortex pair interaction. The standard and symmetric configuration's downstream cross sections returned to roughly axisymmetric after the initial modification was mixed out by turbulence. The beveled and clustered configuration's downstream jet cross sections, on the other hand, remained asymmetric and tended toward triangular and elliptic in shape, respectively. Large amplitude screech tone reductions were identified as a direct result of the drastic cross sectional modifications, with reductions in the $10-25 \mathrm{~dB}$ range achieved at all operating conditions by all of the investigated configurations. A dependence of the reduction on azimuthal orientation was identified, which indicated that shock-vortex interaction was detrimental to reductions. On an azimuthal and operating condition averaged basis, the clustered configuration was identified as the optimal screech reducing configuration with an average of $20 \mathrm{dBs}$ of reduction.

Additionally, reduction in the shock cell spacing was a consequence of the modification of the jet cross section. Through quantitative analysis of streamwise velocity profiles, it was determined that all of the investigated configurations achieved some level of mean shock cell length reduction. Correspondingly, an increase in peak BSAN frequency was achieved by all of the investigated chevron configurations. The theoretical relation of Tam et al. [36] indicated that the trends in shock length reduction and BSAN peak frequency increase were captured using the mean shock length determination routine defined herein. Discrepancies in relative amplitude existed, most likely due to use of non-centerline velocity profiles, which introduced non-orthogonal length measurements because of jet spread. The clustered $\Phi=90^{\circ}$ configuration universally achieved the largest shock length reduction and the consequent largest increase in peak BSAN frequency. 
The average shock strength was also determined from measured velocity profiles and was found to be reduced by all of the chevron configurations. The symmetric configuration was found to have limited azimuthal dependence on shock strength, most likely due to the periodic chevron arrangement. The clustered configuration's $\Phi=90^{\circ}$ plane achieved a lower shock strength than the $\Phi=0^{\circ}$ plane, possibly due to the orientation of the elliptic cross section. The beveled configuration indicated a switch in the optimal shock strength orientation due to a change in the general shock structure. BSAN amplitude reductions roughly trended with the reductions in mean shock strength. The beveled $\Phi=180^{\circ}$ orientation performed optimally in terms of BSAN amplitude reductions at all but the underexpanded condition.

Using the centerline profile of Lau et al. [59] the potential core length of the investigated configurations was calculated. The large amplitude screech of the baseline jet at the $M_{j}=1.36$ and 1.50 conditions resulted in much shorter potential core lengths than predicted for an axisymmetric jet, making reduction conclusions difficult. Confining discussion to the investigated chevron configurations indicated that potential core length reductions were directly configuration dependent, with the clustered configuration achieving the shortest length, followed by the symmetric, beveled and finally standard configurations.

Chevron proximity was identified as a factor which influenced peak near nozzle vorticity magnitude and downstream persistence as it related to vortex interaction. The integrated streamwise vorticity modulus' downstream persistence was shown to correspond to large scale turbulence noise peak amplitude reductions along with reduced aft integrated turbulence values. Consequently, the symmetric configuration achieved the optimum LSS peak amplitude reductions across the investigated operating range, as the reduced chevron proximity enabled the induced vortex pairs to persist further downstream.

Due to the complexity of supersonic jet noise, an optimal noise reducing configuration for all of noise sources across the investigated operating range was not identifiable. In the overexpanded regime the symmetric configuration was able to achieve worst case OASPL reductions within $1 \mathrm{~dB}$ of the standard configuration along with OASPL improvements up to $1.5 \mathrm{~dB}$ in the shock dominated quadrant. At the ideally and under expanded conditions, the beveled configuration at $\Phi=0^{\circ}$ was able to achieve improvements of approximately $2 \mathrm{~dB}$ as compared to the standard configuration. This is an important result as it indicates a possible reduction in thrust penalties of chevrons (reduced number of chevrons) while still achieving appreciable noise reduction.

\section{Acknowledgments}

This work was sponsored by the Office of Naval Research (ONR) through the Jet Noise Reduction (JNR) Project under the Noise Induced Hearing Loss (NIHL) program. The first author would like to thank the Ohio Space Grant Consortium (OSGC) for personal support during this work.

\section{References}

[1] J.M. Seiner, L.S. Ukeiley, B.J. Jansen, C. Kannepalli, S. Dash, Noise reduction technology for f/a-18 e/f aircraft, AIAA Paper 2004-2972, 2004. http://dx.doi. org/10.2514/6.2004-2972.

[2] C. Kuo, J. Veltin, D.K. McLaughlin, Advanced acoustic assessment of small-scale military-style nozzles with chevrons, AIAA Paper 2010-3923, 2010. http://dx.doi.org/10.2514/6.2010-3923.

[3] D. Long, T. McDonald, P. Maye, Effect of inlet flow conditions on noise and performance of supersonic nozzles, AIAA Paper 2010-3920, 2010. http://dx. doi.org/10.2514/6.2010-3920.

[4] B. Henderson, J. Bridges, An MDOE investigation of chevrons for supersonic jet noise reduction, AIAA Paper 2010-3926, 2010. http://dx.doi.org/10.2514/ 6.2010-3926.

[5] J. Bridges, M.P. Wernet, F.C. Frate, PIV measurements of chevrons on F400-series tactical aircraft nozzle model, AIAA Paper 2011-1157, 2011. http://dx. doi.org/10.2514/6.2011-1157.

[6] S. Martens, J.T. Spyropoulos, Practical jet noise reduction for tactical aircraft, American Society of Mechanical Engineers Paper GT2010-23699, 2010, pp. 389-399. http://dx.doi.org/10.1115/GT2010-23699.

[7] R.H. Schlinker, J.C. Simonich, R.A. Reba, Flight effects on supersonic jet noise from chevron nozzles, AIAA Paper 2011-2703, 2011. http://dx.doi.org/10. 2514/6.2011-2703.

[8] D. Munday, N. Heeb, E. Gutmark, J. Liu, K. Kailasanath, Acoustic effect of chevrons on supersonic jets exiting conical convergent-divergent nozzles, AIAA Journal 50 (11) (2012) 2336-2350, http://dx.doi.org/10.2514/1.J051337.

[9] J. Bridges, C.A. Brown, Parametric testing of chevrons on single flow hot jets, AIAA Paper 2004-2824, 2004. http://dx.doi.org/10.2514/6.2004-2824.

[10] V.G. Mengle, U.W. Ganz, E.J. Bultemeier, F.T. Calkins, Clocking effect of chevrons with azimuthally-varying immersions on shockcell/cabin noise, AIAA Paper 2008-3000, 2008. http://dx.doi.org/10.2514/6.2008-3000.

[11] P.S. Tide, K. Srinivasan, Novel chevron nozzle concepts for jet noise reduction, Proceedings of the Institution of Mechanical Engineers, Part G: Journal of Aerospace Engineering 223 (2009) 51-67. http://dx.doi.org/10.1243/09544100JAERO347.

[12] P. Tide, K. Srinivasan, Effect of chevron count and penetration on the acoustic characteristics of chevron nozzles, Applied Acoustics 71 (2010) 201-220, http://dx.doi.org/10.1016/j.apacoust.2009.08.010.

[13] K. Kinzie, S. Martens, D.K. McLaughlin, Supersonic elliptic jet noise: experiments with and without an ejector shroud, AIAA Paper 1993-4349, 1993. http://dx.doi.org/10.2514/6.1993-4349.

[14] K.W. Kinzie, D.K. McLaughlin, An experimental study of noise radiated from supersonic elliptic jets, AIAA Paper 1995-0511, 1995. http://dx.doi.org/10. 2514/6.1995-511.

[15] K.W. Kinzie, D.K. McLaughlin, Aeroacoustic properties of supersonic elliptic jets, Journal of Fluid Mechanics 395 (1999) 1-28, http://dx.doi.org/10.1017/ S002211209900573X.

[16] S.B. Verma, E. Rathakrishnan, Influence of aspect-ratio on the mixing and acoustic characteristics of plain and modified elliptic slot jets, Aerospace Science and Technology 7 (2003) 451-464, http://dx.doi.org/10.1016/S1270-9638(03)00060-9. 
[17] E. Gutmark, K. Schadow, K. Wilson, C. Bicker, Near-field pressure radiation and flow characteristics in low supersonic circular and elliptic jets, Physics of Fluids 31 (9) (1988) 2524-2532, http://dx.doi.org/10.1063/1.867011.

[18] X. Tesson, B.P. Petitjean, D.K. McLaughlin, Experiments on the noise produced by high speed jets with elliptic exhaust nozzles, AIAA Paper 2005-211, 2005. http://dx.doi.org/10.2514/6.2005-211.

[19] K. Viswanathan, Nozzle shaping for reduction of jet noise from single jets, AIAA Journal 43 (2005) 1008-1022, http://dx.doi.org/10.2514/6.2004-2974.

[20] K. Viswanathan, A. Krothapalli, J.M. Seiner, M.J. Czech, B. Greska, B.J. Jansen, Assessment of low-noise nozzle designs for fighter aircraft applications, Journal of Aircraft 48 (2011) 412-423, http://dx.doi.org/10.2514/1.C000285.

[21] M. Samimy, J.H. Kim, J. Kastner, I. Adamovich, Y. Utkin, Active control of high-speed and high-Reynolds-number jets using plasma actuators, Journal of Fluid Mechanics 578 (2007) 305-330, http://dx.doi.org/10.1017/S0022112007004867.

[22] B. Callender, E. Gutmark, R. DiMicco, The design and validation of a coaxial nozzle acoustic test facility, AIAA Paper 2002-0369, 2002. http://dx.doi.org/ $10.2514 / 6.2002-369$.

[23] D. Munday, N. Heeb, E. Gutmark, J. Liu, K. Kailasanath, Supersonic jet noise reduction technologies for gas turbine engines, Journal of Engineering for Gas Turbines and Power 133 (10) (2011) 1-10, http://dx.doi.org/10.1115/1.4002914.

[24] N. Heeb, E. Gutmark, K. Kailasanath, An experimental investigation of the flow dynamics of streamwise vortices of various strength interacting with a supersonic jet, Physics of Fluids 26 (8) (2014) 086102, http://dx.doi.org/10.1063/1.4892008.

[25] D. Munday, E. Gutmark, J. Liu, K. Kailasanath, Flow structure and acoustics of supersonic jets from conical convergent-divergent nozzles, Physics of Fluids 23 (116102) (2011) 1-13, http://dx.doi.org/10.1063/1.3657824.

[26] AIAA, Assessment of experimental uncertainty with application to wind tunnel testing, AIAA Standard S-071A-1999, AIAA, Reston, VA, 1999. URL 〈https://www.aiaa.org/StandardsDetail.aspx?id=3910〉.

[27] AIAA, Assessing experimental uncertainty-supplement to aiaa s-071a-1999, AIAA Guide G-045-2003, AIAA, Reston, VA, 2003. URL <https://www.aiaa. org/StandardsDetail.aspx?id $=3856$.

[28] M.S. Bartlett, Smoothing periodograms from time-series with continuous spectra, Nature 161 (1948) 686-687, http://dx.doi.org/10.1038/161686a0.

[29] H.E. Bass, L.C. Sutherland, A.J. Zuckerwar, D.T. Blackstock, D.M. Hester, Atmospheric absorption of sound: further developments, Journal of the Acoustical Society of America 97 (1) (1994) 680-683, http://dx.doi.org/10.1121/1.412989.

[30] M.J. Lighthill, On sound generated aerodynamically: I. General theory, Proceedings of the Royal Society of London 211 (1107) (1952) 564-587, http://dx. doi.org/10.1098/rspa.1952.0060.

[31] M.B. Alkislar, L.M. Lourenco, A. Krothapalli, Stereoscopic PIV measurements of a screeching supersonic jet, Journal of Visualization 3 (2) (2000) 135-143 , http://dx.doi.org/10.1007/BF03182406.

[32] N.G. Deen, P. Willems, M. Van Sint Annaland, J.A.M. Kuipers, R.G.H. Lammertink, A.J.B. Kemperman, M. Wessling, W.G.J. Van der Meer, On image preprocessing for PIV of single- and two-phase flows over reflecting objects, Experiments in Fluids 49 (2010) 525-530, http://dx.doi.org/10.1007/ s00348-010-0827-y.

[33] Z.R. Carr, K.A. Ahmend, D.J. Forliti, Spatially correlated precision error in digital particle image velocimetry measurements of turbulent flows, Experiments in Fluids 47 (1) (2009) 95-106, http://dx.doi.org/10.1007/s00348-009-0638-1.

[34] E. Lazar, B. DeBlauw, N. Glumac, C. Dutton, G. Elliott, A practical approach to PIV uncertainty analysis, AIAA Paper 2010-4355, 2010. http://dx.doi.org/10. 2514/6.2010-4355.

[35] J.T. Park, A. Derrandji-Aouat, B. Wu, S. Nishio, E. Jacquin, Uncertainty analysis: particle imaging velocimetry, ITTC Recommended Procedures and Guidelines, International Towing Tank Conference, The Japan Society of Naval Architects and Ocean Engineers, Fukuoka, Japan, 2008. URL 〈http://ittc. sname.org/CD\%202011/pdf\%20Procedures\%202011/7.5-01-03-03.pdf).

[36] C.K.W. Tam, J.M. Seiner, J.C. Yu, Proposed relationship between broadband shock associated noise and screech tones, Journal of Sound and Vibration 110 (2) (1986) 309-321, http://dx.doi.org/10.1016/S0022-460X(86)80212-7.

[37] S.P. Pao, J.M. Seiner, Shock-associated noise in supersonic jets, AIAA Journal 21 (5) (1983) 687-693, http://dx.doi.org/10.2514/3.8134.

[38] C. Tam, M. Golebiowski, J.M. Seiner, On the two components of turbulent mixing noise from supersonic jets, AIAA Paper 1996-1716, 1996. http://dx.doi org/10.2514/6.1996-1716.

[39] D.R. Glass, Effects of acoustic feedback on the spread and decay of supersonic jets, AIAA Journal 6 (10) (1968) 1890-1897, http://dx.doi.org/10.2514 3.4897.

[40] J. Bridges, M.P. Wernet, Turbulence associated with broadband shock noise in hot jets, AIAA Paper 2008-2834, 2008. http://dx.doi.org/10.2514/6.20082834.

[41] J. Kastner, B. Malla, E. Gutmark, J. Liu, K. Kailasanath, Using wavelet stochastic estimation to correlate near-field pressure to velocity pod modes in supersonic jet, AIAA Paper 2012-0032, 2012. http://dx.doi.org/10.2514/6.2012-32.

[42] K.C. Massey, K.K. Ahuja, Screech frequency prediction in light of mode detection and convection speed measurements for heated jets, AIAA Paper 19971625, 1997. http://dx.doi.org/10.2514/6.1997-1625.

[43] B. André, T. Castelain, C. Bailly, Broadband shock-associated noise in screeching and non-screeching underexpanded supersonic jets, AIAA Journal 51 (3) (2013) 665-673, http://dx.doi.org/10.2514/1.J052058.

[44] N. Heeb, E. Gutmark, J. Liu, K. Kailasanath, Fluidically enhanced chevrons for supersonic jet noise reduction, AIAA Journal 52 (4) (2014) 799-809, http: //dx.doi.org/10.2514/1.J052508.

[45] J. Panda, An experimental investigation of screech noise generation, Journal of Fluid Mechanics 378 (1999) 71-98, http://dx.doi.org/10.1017/ S0022112098003383.

[46] T.J.S. Jothi, K. Srinivasan, Surface roughness effects on noise from pipe jets, Journal of Sound and Vibration http://dx.doi.org/10.1016/j.jsv.2012.10.001 839-349.

[47] T.D. Norum, J.M. Seiner, Location and propagation of shock associated noise from supersonic jets, AIAA Paper 1980-0983, 1980. http://dx.doi.org/10 2514/6.1980-983.

[48] C. Tam, H.K. Tanna, Shock associated noise of supersonic jets from convergent-divergent nozzles, Journal of Sound and Vibration 81 (1982) 337-358, http://dx.doi.org/10.1016/0022-460X(82)90244-9.

[49] C.W. Kuo, D.K. McLaughlin, P.J. Morris, Effects of supersonic jet conditions on broadband shock-associated noise, AIAA Paper 2011-1032, 2011. http://dx doi.org/10.2514/6.2011-1032.

[50] C.K.W. Tam, Broadband shock-associated noise of moderately imperfectly expanded supersonic jets, Journal of Sound and Vibration 140 (1) (1990) 55-71, http://dx.doi.org/10.1016/0022-460X(90)90906-G.

[51] O. Rask, J. Kastner, E. Gutmark, Understanding how chevrons modify noise in a supersonic jet with flight effects, AIAA Journal 49 (8) (2011) 1569-1576, http://dx.doi.org/10.2514/1.J050628.

[52] K. Gudmundsson, T. Colonius, Spatial stability analysis of chevron jet profiles, AIAA Paper 2007-3599, 2007. http://dx.doi.org/10.2514/6.2007-3599.

[53] B. Malla, D. Cuppoletti, J. Kastner, E. Gutmark, Proper orthogonal decomposition on a subsonic jet exhausted from an axisymmetric nozzle and chevron nozzles with varying penetration, AIAA Paper 2011-278, 2011. http://dx.doi.org/10.2514/6.2011-278.

[54] D. Violato, F. Scarano, Three-dimensional evolution of flow structures in transitional circular and chevron jets, Physics of Fluids 23 (2011) 124104, http: //dx.doi.org/10.1063/1.3665141.

[55] C.K.W. Tam, K.B.M.Q. Zaman, Subsonic jet noise from nonaxisymmetric and tabbed nozzles, AIAA Journal 38 (2000) 592-599, http://dx.doi.org $10.2514 / 2.1029$.

[56] B. Callender, E. Gutmark, S. Martens, Far-field acoustic investigation into chevron nozzle mechanisms and trends, AIAA Journal 43 (1) (2005) 87-95, http://dx.doi.org/10.2514/1.6150. 
[57] M.B. Alkislar, A. Krothapalli, G.W. Butler, The effect of streamwise vortices on the aeroacoustics of a Mach 0.9 jet, Journal of Fluid Mechanics 578 (2007) 139-169, http://dx.doi.org/10.1017/S0022112007005022.

[58] T. Yasunobu, K. Matsuoka, H. Kashimura, S. Matsuo, T. Setoguchi, Numerical study for hysteresis phenomena of shock wave reflection in overexpanded axisymmetric supersonic jet, Journal of Thermal Science 15 (2006) 220-225, http://dx.doi.org/10.1007/s11630-006-0220-6.

[59] J.C. Lau, P.J. Morris, M.J. Fisher, Measurements in subsonic and supersonic free jets using a laser velocimeter, Journal of Fluid Mechanics 93 (1979) 1-27, http://dx.doi.org/10.1017/S0022112079001750.

[60] J.M. Seiner, M.K. Ponton, B. Jansen, N. Lagen, The effects of temperature on supersonic jet noise emission, 14th DGLR/AIAA Aeroacoustics Conference, 1992.

[61] T.D. Norum, J.M. Seiner, Broadband shock noise from supersonic jets, AIAA Journal 20 (1) (1980) 68-73, http://dx.doi.org/10.2514/3.51048.

[62] D.C. Pack, A note on Prandtl's formula for the wavelength of a supersonic gas jet, Quarterly Journal of Mechanics and Applied Mathematics 3 (2) (1950) 173-181, http://dx.doi.org/10.1093/qjmam/3.2.173.

[63] J.E. Ffowcs-Williams, D.L. Hawkings, Sound generation by turbulence and surfaces in arbitrary motion, Philosophical Transactions of the Royal Society of London 264 (1151) (1968) 321-342, http://dx.doi.org/10.1098/rsta.1969.0031.

[64] K.C. Schadow, E. Gutmark, K.J. Wilson, Compressible spreading rates of supersonic coaxial jets, Experiments in Fluids 10 (2-3) (1990) 161-167, http: //dx.doi.org/10.1007/BF00215025.

[65] K.B.M.Q. Zaman, Asymptotic spreading rate of initially compressible jets-experiment and analysis, Physics of Fluids 10 (10) (1998) 2652-2660, http: //dx.doi.org/10.1063/1.869778.

[66] M.O. Burak, L.E. Eriksson, D. Munday, E. Gutmark, E. Prisell, Experimental and numerical investigation of a supersonic c-d chevron nozzle, AIAA Paper 2009-4004, 2009. http://dx.doi.org/10.2514/6.2009-4004. 